\title{
Markov Nets: Probabilistic Models for distributed and concurrent systems
}

\author{
Albert Benveniste, Fellow, IEEE, Eric Fabre, Stefan Haar
}

\begin{abstract}
For distributed systems, i.e., large complex networked systems, there is a drastic difference between a local view and knowledge of the system, and its global view. Distributed systems have local state and time, but do not possess global state and time in the usual sense. In this paper, motivated by the monitoring of distributed systems and in particular of telecommunications networks, we develop a generalization of Markov chains and hidden Markov models (Hmm) for distributed and concurrent systems. By a concurrent system, we mean a system in which components may evolve independently, with sparse synchronizations. We follow a so-called true concurrency approach, in which neither global state nor global time are available. Instead, we use only local states in combination with a partial order model of time. Our basic mathematical tool is that of Petri net unfoldings.
\end{abstract}

Keywords: distributed discrete event systems, Petri nets, probabilistic models, unfoldings.

\section{Motivations}

The difference between a local view and knowledge of a distributed system and its global view is considerable. As an example, it is simply not possible to observe or determine the global state of a telecommunications network. In

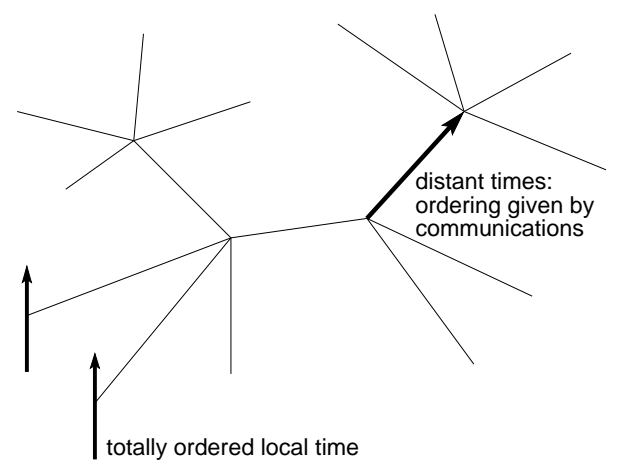

Fig. 1. The structure of time in a networked system.

a networked system, each node possesses its own local state and time. Local time is totally ordered as usual. However, this no longer holds for global time. Sharing time between distant nodes requires explicit synchronization actions and is certainly not instantaneous (see Fig. 1). Hence, events

This paper is dedicated to Alain Bensoussan, for his 60th birthday. This work was supported by the RNRT projects MAGDA and MAGDA2, funded by the Ministère de la Recherche; other partners of the project are France Telecom R\&D, Alcatel, Ilog, and Paris-Nord University.

IRISA/INRIA, Campus de Beaulieu, 35042 Rennes cedex, France. Corresponding author Albert.Benveniste@irisa.fr; this work was started while S.H. was with Ecole normale supérieure, Département d'Informatique / LIENS, 45 Rue d'Ulm, 75230 Paris Cedex 05, and supported by EU project ALAPEDES (TMR). from different nodes are only partially ordered. Similarly, building a global state requires gathering a consistent set of local states, which is not easy (see [27] for this topic). To summarize, networked systems possess local state and totally ordered local time. Global time, however, is only partially ordered, and it is preferred not to consider the global state.

In this paper, motivated by the monitoring of distributed systems and in particular of telecommunications networks, we develop a generalization of stochastic automata, Markov chains, or Hidden Markov Models (HMM), for distributed and concurrent systems having local states and partially ordered time.

As we shall see, a natural model for systems with local states and partially ordered time is that of safe Petri nets. These are introduced in Section II, where the associated structure of runs is also presented, using so-called net unfoldings. Overall, the material of Section II offers nothing new. All this is folklore of computer science but is little known outside. Section III is the core of our contribution. We show how to naturally equip Petri nets with probabilities, in a way compatible with their partial order semantics. In particular, we require that two firing sequences that differ only via their interleaving have identical likelihood. We then introduce Markov nets, a probabilistic extension of Petri nets in which both states and the progress of time are local. Markov nets also have the nice property that, informally said, concurrent choices are stochastically independent. We show that Markov nets satisfy some special kind of Markov property, which appears as the right generalization of the strong Markov property for classical HMM's or Markov chains. Our construction does not work for general Petri nets, however. It requires some structural conditions that are in particular satisfied by free choice nets, but are in fact more general. Handling general (safe) Petri nets is investigated in section IV. Related work is discussed in Section V. Finally, Section VI outlines some conclusions and perspectives.

Due to lack of space, some interesting aspects are omitted, and the reader is referred to an earlier extended version of this paper [1].

\section{Petri NETS AND UNFOldings, AS PARTIAL ORDER MODELS OF DISTRIBUTED SYSTEMS}

\section{A. From concurrent automata to partial order models and Petri nets: an informal discussion}

In this subsection we discuss informally why Petri nets are an adequate framework to model distributed systems with local states and partial order model of time. We con- 
sider automata $\mathcal{A}=\left(X, \Sigma, \delta, x_{0}\right)$, where $X$ and $\Sigma$ are the sets of states and events, $\delta$ is the transition relation, and $x_{0}$ is the initial state. Two such automata $\mathcal{A}^{\prime}$, resp. $\mathcal{A}^{\prime \prime}$, can either perform private actions, or can synchronize by emitting identical labels belonging to $\Sigma^{\prime \prime} \cap \Sigma^{\prime}$. This is illustrated in Fig. 2 where we show a run for a pair $\mathcal{A}^{\prime} \times \mathcal{A}^{\prime \prime}$

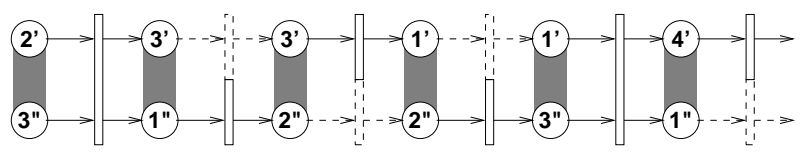

Fig. 2. A run for a pair $\mathcal{A}^{\prime} \times \mathcal{A}^{\prime \prime}$ of concurrent automata.

of concurrent automata. In this figure, local states are represented by circles (with their names, primed and double primed for the first and second automaton, respectively) and transitions are shown as rectangles (their labels are omitted). States of $\mathcal{A}^{\prime} \times \mathcal{A}^{\prime \prime}$ are pairs of local states, depicted by two local states linked by a grey zone. Arrows and rectangles can be solid or dashed. Dashed arrows and rectangles depict "silent" transitions, in which the considered automaton does not change its state and emits nothing (these dummy transitions have only the purpose of letting the other automaton progress). Solid arrows and rectangles depict effective transitions in which some move is performed and a label is emitted. A long rectangle indicates a synchronizing transition. Between their synchronizing transitions, the two components evolve independently and concurrently, and therefore it is advisable not to distinguish between the above run and the one shown in Fig. 3, as these differ only in the way concurrent transitions in-

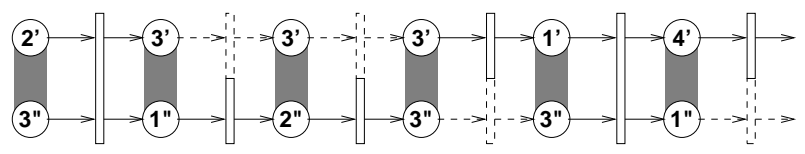

Fig. 3. A different interleaving of the run of Fig. 2.

terleave. This defines, informally, an equivalence relation on times. Therefore, in randomizing runs of automata, one should rather randomize equivalence classes modulo the above equivalence relation, not the runs themselves.

Hence the alternative picture for the runs of our concurrent automata, shown in Fig. 4, should be preferred instead. In this picture, transitions are not linearly ordered

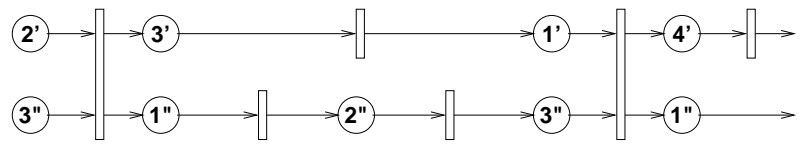

Fig. 4. A common partial oder view of the two runs of Fig. 2 and Fig. 3.

any more as a global sequence of events, and the grey zones indicating global states have disappeared. Instead, states become local and events are only partially ordered as specified by the bipartite directed graph shown. A quick examination of this figure reveals that the right way to represent transitions should be in fact the one shown in Fig. 5.

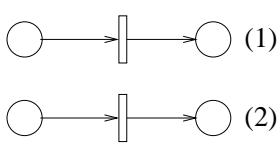

(3)

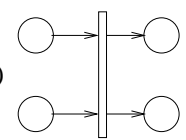

Fig. 5. The generic transitions of Fig. 4 .

Transition of type (1) depicts a generic private transition of $\mathcal{A}^{\prime}$, transition of type (2) depicts a generic private transition of $\mathcal{A}^{\prime \prime}$, and transition of type (3) depicts a generic synchronizing transition of $\mathcal{A}^{\prime}$ and $\mathcal{A}^{\prime \prime}$. But transition of type (3) can be regarded as a transition of a Petri net, with its pre- and post-set of places.

Extending this discussion to several concurrent automata, we naturally arrive at considering Petri nets [9], [28] instead of automata and their products ${ }^{1}$. For our purpose, the important facts about nets are the following: 1/ states are local; referring to our example above, the final picture involves only states $x^{\prime}$ and $x^{\prime \prime}$ of the components, but not states of the product automaton $\mathcal{A}^{\prime} \times \mathcal{A}^{\prime \prime}$, which are pairs $\left(x^{\prime}, x^{\prime \prime}\right) ; 2 /$ time is local too, as it is only partially ordered; referring to our example above, each component has a totally ordered time, but the global system has not.

Running example: stating our objectives. Fig.6-left introduces our running example, which is discussed in de-
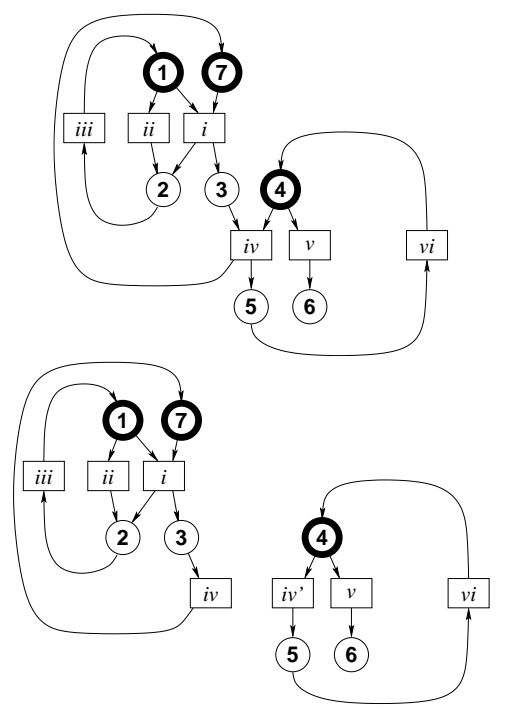

Fig. 6. Running example (top), and its split version (bottom). Routing choices occur at places 1 and 4 .

tail in [8] to illustrate asynchronous fault diagnosis of discrete event systems. It represents the evolution of the failed/safe status of two components interacting via their shared places 3,7 . The first component possesses places $1,2,3,7$ and transitions $i, i i, i i i$ and the second component possesses places 3,7,4,5,6 and transitions iv, v,vi. Although simple, this example is meaningful and rich. Thick circles

\footnotetext{
${ }^{1}$ More precisely, we consider Petri nets as a model of distributed automata, i.e., nets in which the status of a place is boolean-the only token is either absent or present. This is known as safe Petri nets.
} 
indicate an initial token. Our objective is twofold:

to equip with a probability the set of all runs, seen as partial orders,

by only randomizing routing choices, locally.

Now, Fig. 6-right shows a split version of the former, in which transition $i v$ has been duplicated; this results in a Petri net made of two noninteracting components. For this case, we shall require that its two components are probabilistically independent:

probabilistic independence shall respect concurrency.

Discussing some previous approaches relating Petri nets and probabilities. Note first that requirements $(1,2)$ are not suited to models involving physical time. The latter are frequently used (with good reasons) for performance evaluation studies. Stochastic timed Petri net models for performance evaluation belong to these class. They give raise to Markov chains which do not obey our above requirements. In fact, for timed models, the physical time itself is global and results in an implicit coupling between all components, should they interact or not. In our work we consider untimed models, because the timing information regarding the events collected at a network supervisor is not accurate enough to fix nondeterminism due to interleaving (requirement(1)). And we want to prepare for distributed supervision: two supervisors dealing with noninteracting domains should be allowed to ignore each other (requirement (2)).

A detailed discussion of this topic is found in [2], we give only an outline here. Randomizing Petri nets is performed, for Stochastic Petri nets (SPN) [21][6], by applying race policies in which exponential waiting times are allocated to enabled transitions and only the earliest one fires. The use of race policies involves global time and violates our previous two requirements. As an alternative, we can choose to fire only maximal sets of enabled transitions. This way, Petri net executions become independent from the nondeterminism due to the interleaving of concurrent transitions in a firing sequence. Unfortunately, such a policy is not local, and does not satisfy our second requirement. Other approaches have been proposed as well. However, as explained in [2], Generalized Stochastic Petri nets (GSPN) [3][4] and Probabilistic Petri nets (PPN) [10] do not satisfy these requirements either. In this paper, we develop an approach that meets requirements $(1,2)$.

\section{B. Background notions on Petri nets and their unfoldings}

Basic references are [28][9][11]. Homomorphisms, conflict, concurrency, and unfoldings, are the essential concepts on which a true concurrency and fully asynchronous view of Petri nets is based. In order to introduce these notions, it will be convenient to consider general "nets" in the sequel.

Nets, homomorphisms, and labelings. A net is a triple $\mathcal{N}=(P, T, \rightarrow)$, where $P$ and $T$ are disjoint sets of places and transitions, and $\rightarrow \subseteq(P \times T) \cup(T \times P)$ is the flow relation. The reflexive transitive closure of the flow relation $\rightarrow$ is denoted by $\preceq$, and its irreflexive transitive closure is denoted by $\prec$. Places and transitions are called nodes, generically denoted by $x$. For $x \in P \cup T$, we denote by ${ }^{\bullet} x=\{y: y \rightarrow x\}$ the preset of node $x$, and by $x^{\bullet}=\{y: x \rightarrow y\}$ its postset. For $X \subset P \cup T$, we write $\bullet X=\bigcup_{x \in X}^{\bullet} x$ and $X^{\bullet}=\bigcup_{x \in X} x^{\bullet}$. A homomorphism from a net $\mathcal{N}$ to a net $\mathcal{N}^{\prime}$ is a map $\varphi: P \cup T \mapsto P^{\prime} \cup T^{\prime}$ such that: $1 / \varphi(P) \subseteq P^{\prime}, \varphi(T) \subseteq T^{\prime}$, and $2 /$ for every transition $t$ of $\mathcal{N}$, the restriction of $\varphi$ to ${ }^{\bullet} t$ is a bijection between ${ }^{\bullet} t$ and ${ }^{\bullet} \varphi(t)$, and the restriction of $\varphi$ to $t^{\bullet}$ is a bijection between $t^{\bullet}$ and $\varphi(t)^{\bullet}$. For $\mathcal{N}=(P, T, \rightarrow)$ a net, a labeling is a map $\lambda: T \mapsto A$, where $A$ is some finite alphabet. A net $\mathcal{N}=(P, T, \rightarrow, \lambda)$ equipped with a labeling $\lambda$ is called a labeled net.

Occurrence nets, conditions and events. Two nodes $x, x^{\prime}$ of a net $\mathcal{N}$ are in conflict, written $x \# x^{\prime}$, if there exist distinct transitions $t, t^{\prime} \in T$, such that ${ }^{\bullet} t \cap{ }^{\prime} \neq \emptyset$ and $t \preceq x, t^{\prime} \preceq$ $x^{\prime}$. A node $x$ is in self-conflict if $x \# x$. An occurrence net is a net $\mathcal{O}=(B, E, \rightarrow)$, satisfying the following additional properties:

$$
\begin{aligned}
& \forall x \in B \cup E: \neg[x \# x] \quad \text { (no node is in self-conflict) } \\
& \forall x \in B \cup E: \neg[x \prec x] \quad(\preceq \text { is a partial order }) \\
& \forall x \in B \cup E:|\{y: y \prec x\}|<\infty \quad(\preceq \text { is well founded }) \\
& \forall b \in B:|\bullet b| \leq 1 \quad \text { (each place has at most }
\end{aligned}
$$

We will assume that the set of minimal nodes of $\mathcal{O}$ is contained in $B$, and we denote by $\min (B)$ or $\min (\mathcal{O})$ this minimal set. Specific terms are used to distinguish occurrence nets from general nets. $B$ is the set of conditions, $E$ is the set of events, $\prec$ is the causality relation. Nodes $x$ and $x^{\prime}$ are concurrent, written $x \Perp x^{\prime}$, if neither $x \preceq x^{\prime}$, nor $x^{\prime} \preceq x$, nor $x \# x^{\prime}$ hold. A co-set is a set $c$ of concurrent conditions. A maximal (for set inclusion) co-set is called a cut. A configuration $\kappa$ is a sub-net of $\mathcal{O}$, which is conflict-free (no two nodes are in conflict), causally closed (if $x^{\prime} \preceq x$ and $x \in \kappa$, then $\left.x^{\prime} \in \kappa\right)$, and contains $\min (\mathcal{O})$.

Occurrence nets are useful to represent executions of Petri nets. They form a subclass of nets in which essential properties are visible via the topological structure of the bipartite graph.

Petri nets. For $\mathcal{N}$ a net, a marking of $\mathcal{N}$ is a multiset $M$ of places, i.e., a map $M: P \mapsto\{0,1,2, \ldots\}$. A Petri net is a pair $\mathcal{P}=\left(\mathcal{N}, M_{0}\right)$, where $\mathcal{N}$ is a net having finite sets of places and transitions, and $M_{0}$ is an initial marking. A transition $t \in T$ is enabled at marking $M$ if $M(p)>0$ for every $p \in{ }^{\bullet} t$. Such a transition can fire, leading to a new marking $M^{\prime}=M-{ }^{\bullet} t+t^{\bullet}$; we denote this by $M[t\rangle M^{\prime}$. The set of reachable markings of $\mathcal{P}$ is the smallest (w.r.t. set inclusion) set $M_{0}[\rangle$ containing $M_{0}$ and such that $M \in$ $M_{0}[\rangle$ and $M[t\rangle M^{\prime}$ together imply $M^{\prime} \in M_{0}[\rangle$. Petri net $\mathcal{P}$ is safe if $M(P) \subseteq\{0,1\}$ for every reachable marking $M$. Throughout this paper, we consider only safe Petri nets, hence marking $M$ can be regarded as a subset of places. A finite occurrence net $\mathcal{B}$ can be regarded as a Petri net, where the initial marking is $M_{0}=\min (\mathcal{B})$. 
In this paper, we restrict ourselves to the class of safe nets satisfying the additional condition: for every transition $t$, $\bullet t \neq \emptyset \wedge t^{\bullet} \neq \emptyset$. Note that a safe Petri net such that each transition has one place in its preset and one in its postset, can be seen as an automaton.

Branching processes and unfoldings. A branching process of Petri net $\mathcal{P}$ is a pair $\mathcal{B}=(\mathcal{O}, \varphi)$, where $\mathcal{O}$ is an occurrence net, and $\varphi$ is a homomorphism from $\mathcal{O}$ to $\mathcal{P}$ regarded as nets, such that: $1 /$ the restriction of $\varphi$ to $\min (\mathcal{O})$ is a bijection between $\min (\mathcal{O})$ and $M_{0}$ (the set of initially marked places), and $2 /$ for all $e, e^{\prime} \in E,{ }^{\bullet} e=e^{\prime}$ and $\varphi(e)=\varphi\left(e^{\prime}\right)$ together imply $e=e^{\prime}$. By abuse of notation, we shall sometimes write $\min (\mathcal{B})$ instead of $\min (\mathcal{O})$. For $\mathcal{B}$ a branching process of $\mathcal{P}$, and $e$ an event not belonging to $\mathcal{B}$ but having its preset contained in $\mathcal{B}$, we call $e$ a possible continuation of $\mathcal{B}$, written $\mathcal{B} \odot e$. Furthermore, we denote the corresponding extended branching process by $\mathcal{B} \bullet e$, and call it an extension of $\mathcal{B}$.

The set of all branching processes of Petri net $\mathcal{P}$ is uniquely defined, up to an isomorphism (i.e., a renaming of the conditions and events), and we shall not distinguish isomorphic branching processes. For $\mathcal{B}, \mathcal{B}^{\prime}$ two branching processes, $\mathcal{B}^{\prime}$ is a prefix of $\mathcal{B}$, written $\mathcal{B}^{\prime} \sqsubseteq \mathcal{B}$, if there exists an injective homomorphism $\psi$ from $\mathcal{B}^{\prime}$ into $\mathcal{B}$, such that $\psi\left(\min \left(\mathcal{B}^{\prime}\right)\right)=\min (\mathcal{B})$, and the composition $\varphi \circ \psi$ coincides with $\varphi^{\prime}$, where $\circ$ denotes the composition of maps.

By theorem 23 of [13], there exists (up to an isomorphism) a unique maximal branching process with respect to $\sqsubseteq$, we call it the unfolding of $\mathcal{P}$, and denote it by $\Omega_{\mathcal{P}}$. The unfolding of $\mathcal{P}$ possesses the following universal property. For every occurrence net $\mathcal{O}$, and every homomorphism $\phi: \mathcal{O} \mapsto \mathcal{P}$, there exists an injective homomorphism $\iota: \mathcal{O} \mapsto \Omega_{\mathcal{P}}$, such that $\phi=\varphi \circ \iota$, where $\varphi$ denotes the homomorphism associated to $\Omega_{\mathcal{P}}$; this decomposition expresses that $\Omega_{\mathcal{P}}$ "maximally unfolds" $\mathcal{P}$. If $\mathcal{P}$ is itself an occurrence net and $M_{0}=\min (\mathcal{P})$ holds, then $\Omega_{\mathcal{P}}$ identifies with $\mathcal{P}$.

Configurations of the unfolding $\Omega_{\mathcal{P}}$ are adequate representations of the firing sequences of $\mathcal{P}$. Let $M_{0}, M_{1}, M_{2}, \ldots$ be a maximal firing sequence of $\mathcal{P}$, and let $M_{k-1}\left[t_{k}\right\rangle M_{k}$ be the associated sequence of fired transitions. Then there exists a unique maximal (for set inclusion) configuration $\kappa$ of $\Omega_{\mathcal{P}}$ having the following properties: $\kappa$ is the union of a sequence $e_{1}, e_{2}, \ldots$ of events and a sequence $\mathbf{c}_{0}, \mathbf{c}_{1}, \mathbf{c}_{2}, \ldots$ of cuts, such that, for each $k>0, \varphi\left(\mathbf{c}_{k}\right)=M_{k}, \varphi\left(e_{k}\right)=t_{k}$, and $\mathbf{c}_{k-1} \supseteq \bullet_{k}, e_{k}^{\bullet} \subseteq \mathbf{c}_{k}$. Conversely, each maximal configuration of $\Omega_{\mathcal{P}}$ defines a maximal firing sequence, which is unique up to the interleaving of consecutive structurally independent transitions - transitions $t$ and $t^{\prime}$ are structurally concurrent iff $\bullet t^{\prime} \cap\left(\bullet t \cup t^{\bullet}\right)=\emptyset$ and $\left.\bullet t \cap\left(\bullet t^{\prime} \cup t^{\prime}\right)^{\bullet}\right)=\emptyset$.

Maximal configurations of $\Omega_{\mathcal{P}}$ are called runs of $\mathcal{P}$ and are generically denoted by $\omega$ (and sometimes by $v$ or $w$ ). By abuse of notation, we write $\omega \in \Omega_{\mathcal{P}}$ to express that $\omega$ is a run of $\mathcal{P}$.

Running example, continued. Fig. 7 shows again our running example. The Petri net $\mathcal{P}$ is shown on the top left. Its places are $1,2,3,4,5,6,7$, and its transitions are

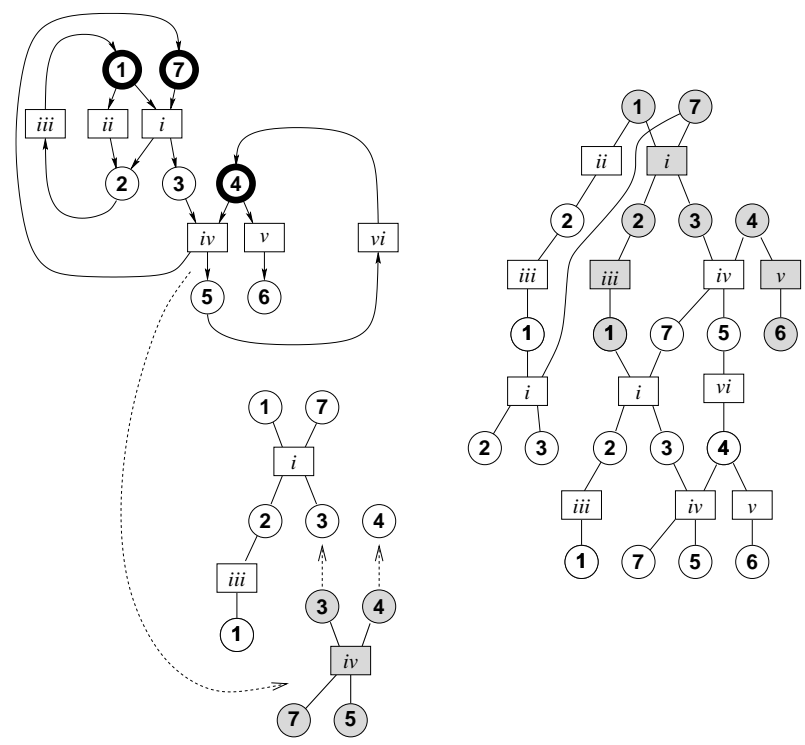

Fig. 7. Running example (top left), a configuration (bottom left), and a branching process (right). For this and subsequent examples, we take the following convention for drawing Petri nets and occurrence nets. In Petri nets, the flow relation is depicted using directed arrows. In occurrence nets, since no cycle occurs, the flow relation progresses downwards, and therefore there is no need to figure them via directed arrows, standard solid lines are used instead.

$i, i i, i i i, i v, v, v i$. Places constituting the initial marking are encircled in thick. A branching process $\mathcal{B}=(\mathcal{O}, \varphi)$ of $\mathcal{P}$ is shown on the right. Its conditions are depicted by circles, and its events are figured by boxes. Each condition $b$ of $\mathcal{B}$ is labeled by the place $\varphi(b)$ of $\mathcal{P}$. Each event $e$ of $\mathcal{B}$ is labeled by the transition $\varphi(e)$ of $\mathcal{P}$. A configuration of Petri net $\mathcal{P}$ is shown in grey. Note that the minimal condition labeled by 7 is branching in $\mathcal{B}$, although it is not branching in $\mathcal{P}$ itself. The reason is that, in $\mathcal{P}$, the token can freely move along the circuit $1 \rightarrow i i \rightarrow 2 \rightarrow i i i \rightarrow 1$, and resynchronize afterwards with the token sitting in 7 . The mechanism for constructing the unfolding of Petri net $\mathcal{P}$ is illustrated in the bottom left, it is informally explained as follows. Put the three conditions labeled by the initial marking of $\mathcal{P}$, this is the minimal branching process of $\mathcal{P}$. Then, for each constructed branching process $\mathcal{B}$, select a co-set $c$ of $\mathcal{B}$, which is labeled by the preset ${ }^{\bullet} t$ of some transition $t$ of $\mathcal{P}$, and has no event labeled by $t$ in its postset within $\mathcal{B}$. Append to $c$ a net isomorphic to $\bullet t \rightarrow t \rightarrow t^{\bullet}$ (recall that $\varphi(\bullet t)=c$ ), and label its additional nodes by $t$ and $t^{\bullet}$, respectively. Performing this recursively yields all possible finite branching processes of $\mathcal{P}$. Their union is the unfolding $\Omega_{\mathcal{P}}$.

Comparison with automata and their trajectories. Fig. 8 shows an automaton (top left, the initial state indicated by an ingoing arrow), its translation as a Petri net (bottom left, the initial marking is composed of the place filled in grey), and a branching process of the so obtained Petri net (right). Since each transition in the Petri net has a single place in its pre- and postset, the unfolding is a tree. Each maximal directed path of this tree represents a run of the automaton. 


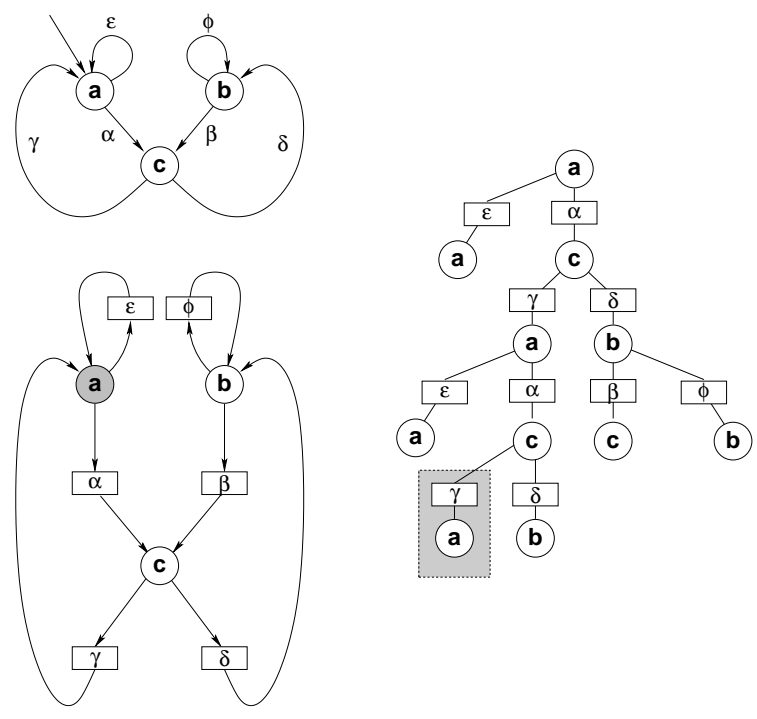

Fig. 8. Showing a branching process of an automaton (i.e., a prefix of its unfolding).

\section{Adding PRobabilities: Markov nets}

This section is the core of the paper. We introduce Markov nets by equipping unfoldings with probabilities.

\section{A. Markov nets}

We introduce Markov nets by locally randomizing the possible choices, for each individual branching place of the considered Petri net. To avoid uninteresting technicalities, we shall consider that the initial marking is fixed, and not randomized. Extending our theory and results to the more general case of random initial marking is easy (we need to deal with a collection of unfoldings, one for each different initial marking).

Definition 1 (Markov net) We consider a Petri net $\mathcal{P}=$ $\left(P, T, \rightarrow, M_{0}\right)$. Let $P_{c}$ be the set of those $p \in P$ whose post-set $p^{\bullet}$ contains at least two transitions - we say that $p$ exhibits a choice, or, equivalently, that $p$ is branching. Let $\pi$ be a transition probability from $P_{c}$ to $T$, i.e., a set $\pi \triangleq\left(\pi_{p}\right)_{p \in P_{c}}$, where $\pi_{p}$ is a probability over $p^{\bullet}$. The pair $\mathcal{M}=(\mathcal{P}, \pi)$ is called a Markov net, and $\pi$ is called its routing policy, it specifies the probability distribution of routings - for convenience, we shall sometimes write $\pi(t \mid p)$ instead of $\pi_{p}(t)$, to reflect that $\pi_{p}$ plays the role of a conditional probability.

Referring to Fig. 9, $P_{c}$ is composed of the places filled in dark grey, and routing policies $\pi_{1}, \pi_{4}$ are shown on the Petri net. Note that the minimal condition labeled by 7 in the unfolding is not a choice condition, since place 7 in the Petri net does not belong to $P_{c}$. In fact, the branching at the minimal condition labeled by 7 in the unfolding does not correspond to choice, and should not be randomized. The remainder of this section is mainly devoted to the construction of the probability of runs for Markov nets, according to the partial order semantics.
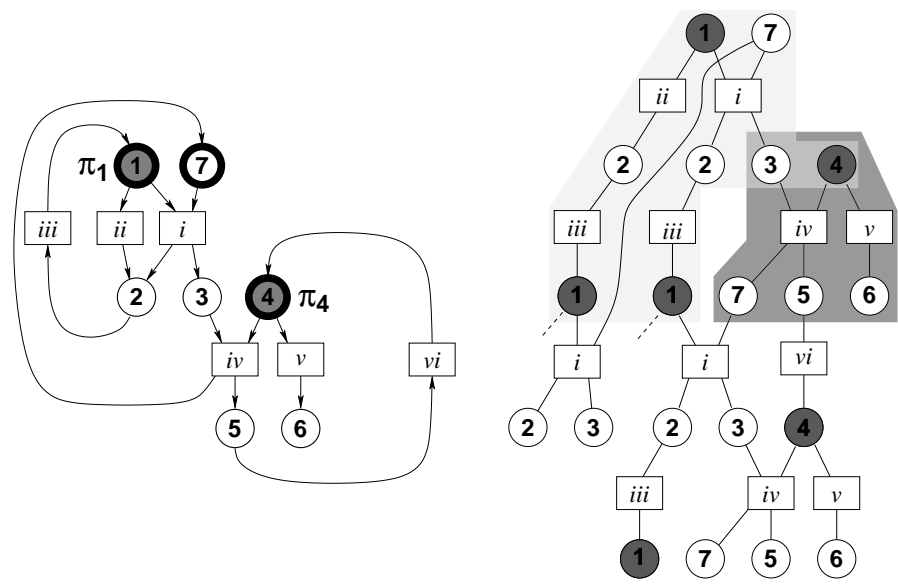

Fig. 9. Markov net: illustrating routing policy, stopping time, layer. (The mid-grey area comprising the conditions labeled by 3,4 indicates the superposition of the two light- and dark-grey areas.)

In the extended paper [1], we also consider Markov nets with random labels, and we propose a definition of the product of (labeled) Markov nets, denoted by $\mathcal{M}^{\prime} \times \mathcal{M}^{\prime \prime}$. It consists in equipping the product $\mathcal{P}^{\prime} \times \mathcal{P}^{\prime \prime}$ of the underlying Petri nets with proper routing policies. It has the important desirable feature that, if the underlying Petri nets $\mathcal{P}^{\prime}$ and $\mathcal{P}^{\prime \prime}$ do not interact (i.e., share no transition), then the product $\mathcal{M}^{\prime} \times \mathcal{M}^{\prime \prime}$ makes the two components $\mathcal{M}^{\prime}$ and $\mathcal{M}^{\prime \prime}$ independent in the probabilistic sense, see Theorem 2 in Section III-E.

\section{B. Branching processes and stopping times}

When equipping sets of runs with probabilities, the first issue to be addressed is the following: which $\sigma$-algebras should one use? In classical theories of stochastic processes, there is typically an increasing family of $\sigma$-algebras $\left(\mathcal{F}_{n}\right)$, indexed by some totally ordered time index, say $n \in \mathbf{N}$. Then, stopping times are considered to model "causal" random instants, they can be used to index $\sigma$-algebras as well. What are the counterparts of these classical notions in our case?

The case of automata (see Fig. 8). In automata, the progress of time coincides with the occurrence of one transition in the automaton. Let $\tau$ be a stopping time, i.e., an integer valued random variable such that deciding whether or not $\tau \leq n$ requires at most $n$ successive transitions of the automaton. For $\omega$ a run, denote by $\omega_{n}$ the prefix of $\omega$ of length $n$. Since $\tau$ is a stopping time, deciding whether or not $\tau=n$ requires $n$ successive transitions of the automaton. Thus $\omega_{\tau} \triangleq \omega_{\tau(\omega)}$ is well defined. Then denote by $\mathcal{B}_{\tau}$ the union of all $\omega_{\tau}$, for $\omega$ running over the set of all runs: $\mathcal{B}_{\tau}$ is a branching process. The following lemma relates stopping times to branching processes, for automata.

Lemma 1: Assume that $\mathcal{P}$ is an automaton. Then, a branching process $\mathcal{B}$ of $\mathcal{P}$ has the form $\mathcal{B}_{\tau}$ for some stopping time $\tau$ iff it satisfies the following property: $\forall b \in B$ such that $\varphi(b) \in P_{c}$, either $b_{\mathcal{B}}^{\bullet}=\emptyset$ or $b_{\mathcal{B}}^{\bullet}=b^{\bullet}$, where $b_{\mathcal{B}}^{\bullet}$ denotes the postset of condition $b$ in $\mathcal{B}$ (and $b^{\bullet}$ denotes, as usual, the postset of condition $b$ in the whole unfolding $\left.\Omega_{\mathcal{P}}\right)$. $\diamond$ 
In other words, stopping times coincide with branching processes such that all branching conditions have their postset either entirely inside the process or entirely outside of it.

Proof: See the Appendix (the entire branching process $\mathcal{B}$ shown in Fig. 8 possesses this property, but the branching process $\mathcal{B}^{\prime}$ obtained by removing the shaded subnet does not.)

The case of general Petri nets (cf. Fig. 9). Lemma 1 suggests the proper notion of stopping time for general Petri nets:

Definition 2 (stopping time) A branching process $\mathcal{B}=$ $(B, E, \rightarrow, \varphi)$ is called a stopping time if it satisfies the following condition: $\forall b \in B$ such that $\varphi(b) \in P_{c}$, either $b_{\mathcal{B}}^{\bullet}=\emptyset$ or $b_{\mathcal{B}}^{\bullet}=b^{\bullet}$, where $b_{\mathcal{B}}^{\bullet}$ denotes the postset of condition $b$ in $\mathcal{B}$.

Lemma 2: The set of stopping times is stable under arbitrary intersections and unions.

$$
\text { Proof: Obvious. }
$$

We are now ready to introduce our concept of layer, as a formalization of atomic progress of time, from one stopping time to a successor.

Definition 3 (layer) Let $\mathcal{B}$ and $\mathcal{B}^{\prime}$ be two stopping times such that $1 / \mathcal{B}^{\prime}$ is strictly contained in $\mathcal{B}$, and $2 /$ there exists no stopping time strictly containing $\mathcal{B}^{\prime}$ and strictly contained in $\mathcal{B}$. We call a layer the following suffix of $\mathcal{B}$ :

$$
L=\left(\mathcal{B} \backslash \mathcal{B}^{\prime}\right) \cup \bullet\left(\mathcal{B} \backslash \mathcal{B}^{\prime}\right)
$$

where $\cup$ denotes the union of labelled graphs.

This notion is illustrated in Fig. 9, where the subnet contained in the dark grey area and having the conditions labeled by 3,4 as minimal conditions, is a layer.

Decomposition (3) is not unique. However, if decomposition $L=\left(\mathcal{B}_{i} \backslash \mathcal{B}_{i}^{\prime}\right) \cup \bullet\left(\mathcal{B}_{i} \backslash \mathcal{B}_{i}^{\prime}\right), i=1,2$ holds, then it also holds with $\mathcal{B}_{1} \cap \mathcal{B}_{2}$ and $\mathcal{B}_{1}^{\prime} \cap \mathcal{B}_{2}^{\prime}$ in lieu of $\mathcal{B}_{i}$ and $\mathcal{B}_{i}^{\prime}$, $i=1,2$. Hence the set of pairs $\left(\mathcal{B}, \mathcal{B}^{\prime}\right)$ for which decomposition (3) holds has a unique minimal pair, we take this pair as the canonical decomposition of layer $L$, and we write this canonical decomposition as follows:

$$
L=\mathcal{B} / \mathcal{B}^{\prime}
$$

The set of all layers of the considered unfolding is denoted by $\mathcal{L}$. Now we collect some useful properties of $\mathcal{L}$. Let $B_{c}$ denote the set of conditions that are branching in both $\mathcal{P}$ and $\Omega_{\mathcal{P}}$, we call them branching conditions in the sequel (for $A$ a set, $|A|$ denotes its cardinal):

$$
B_{c}=\left\{b \in B:\left|b^{\bullet}\right|>1 \wedge \varphi(b) \in P_{c}\right\} .
$$

\section{Lemma 3:}

1. Consider the following relation on $\mathcal{L}$ :

$$
L^{\prime} \prec L \quad \text { iff } \quad L=\mathcal{B} / \mathcal{B}^{\prime} \text { and }\left(L^{\prime} \cap B_{c}\right) \subseteq \mathcal{B}^{\prime} .
$$

Then $(\mathcal{L}, \prec)$ is a partial order.

2. Any stopping time decomposes as a union of layers having pairwise disjoint sets of events. Such a decomposition is unique, it defines a bijection between stopping times and prefixes of $(\mathcal{L}, \prec)$.

$$
\text { Proof: Obvious. }
$$

In the sequel, we identify $\mathcal{L}$ with the directed acyclic graph (DAG) defined as follows: for $L, L^{\prime} \in \mathcal{L}$, write $L \rightarrow L^{\prime}$ iff $L \prec L^{\prime}$ and $L \cap L^{\prime} \neq \emptyset$ (meaning that layers $L$ and $L^{\prime}$ are neighbours in the unfolding).

\section{Equipping unfoldings of Petri nets with probabilities \\ C.1 Choice-compact Petri nets.}

In equipping unfoldings of Petri nets with probabilities, we are faced with two types of difficulties:

1. To obtain probability measures on unfoldings, we naturally think of using the labelling map $\varphi$ in order to lift the routing policy $\pi$ from Petri net $\mathcal{P}$ to its unfolding $\Omega_{\mathcal{P}}$. Let us make an attempt by defining $\pi_{\Omega}(e \mid b)=\pi(\varphi(e) \mid \varphi(b))$, for $e \in b^{\bullet}$. This is a sound definition if the map $e \mapsto \varphi(e)$, from $b^{\bullet}$ to $\varphi(b)^{\bullet}$, is injective. In this case $\pi_{\Omega}$ becomes a positive measure with total mass $\leq 1$, it is a probability if $e \mapsto \varphi(e)$ is bijective. If $e \mapsto \varphi(e)$ is not injective, we are in trouble lifting $\pi(. \mid \varphi(b))$ to a transition probability from $b$ to its postset. Now, check carefully the definition of branching processes in subsection II-B: it is stated that, for $t=\varphi(e)$, the two subnets $\bullet e \rightarrow e \rightarrow e^{\bullet}$ and $\bullet^{\bullet} t \rightarrow t \rightarrow t^{\bullet}$, seen as directed graphs, are isomorphic. But such a property does not hold for conditions and their pre- and postsets. This motivates the following definition of choice-conformal branching processes, namely branching processes satisfying the following condition: for all $b \in B$ such that $\varphi(b) \in P_{c}$, the restriction to $b^{\bullet}$ of the labelling map $e \mapsto \varphi(e)$ is injective, from $b^{\bullet}$ into $\varphi(b)^{\bullet}$.

2. The second problem is that of the finiteness of layers. In constructing our probability distribution on $\Omega_{\mathcal{P}}$, we need to apply a Kolmogorov extension argument, by constructing a "consistent" family of probabilities, one for each finite stopping time. Progressing from one stopping time to a next one proceeds by concatenating some layer, seen as an atomic progress of the random process under construction. The idea is to lift our routing policy $\pi$ to a transition probability on the layer in consideration. This is easy if the considered layer involves only finitely many routings.

The above two points motivate the following definition:

Definition 4 (choice-compact) $\mathcal{P}=\left(P, T, \rightarrow, M_{0}\right)$ is called choice-compact iff it satisfies the following two conditions:

1. Choice-conformalness: For each condition $b \in B_{c}$, the restriction to $b^{\bullet}$ of the labelling map $e \mapsto \varphi(e)$ is injective, from $b^{\bullet}$ into $\varphi(b)^{\bullet}$.

2. Finiteness of layers: All layers of $\Omega_{\mathcal{P}}$ possess only finitely many conditions that are labelled by places belonging to $P_{c}$.

Examples and counter-examples. Our running example of Fig. 9 possesses a choice-conformal unfolding. The reason is that places belonging to $P_{c}$ (places 1,4 in Fig. 7-top left), 
and places causing branching in the unfolding due to synchronization (place 7 in Fig. 7 -top left), are disjoint. Ancillary synchronizations and active choices occur in distinct sets of places. Also, our running example possesses finite layers. In fact, all layers are isomorphic to one of the two layers shown in this figure, in light and mid grey, respectively. Thus, our running example is choice-compact.

Now, Fig. 10 shows an example of a Petri net which
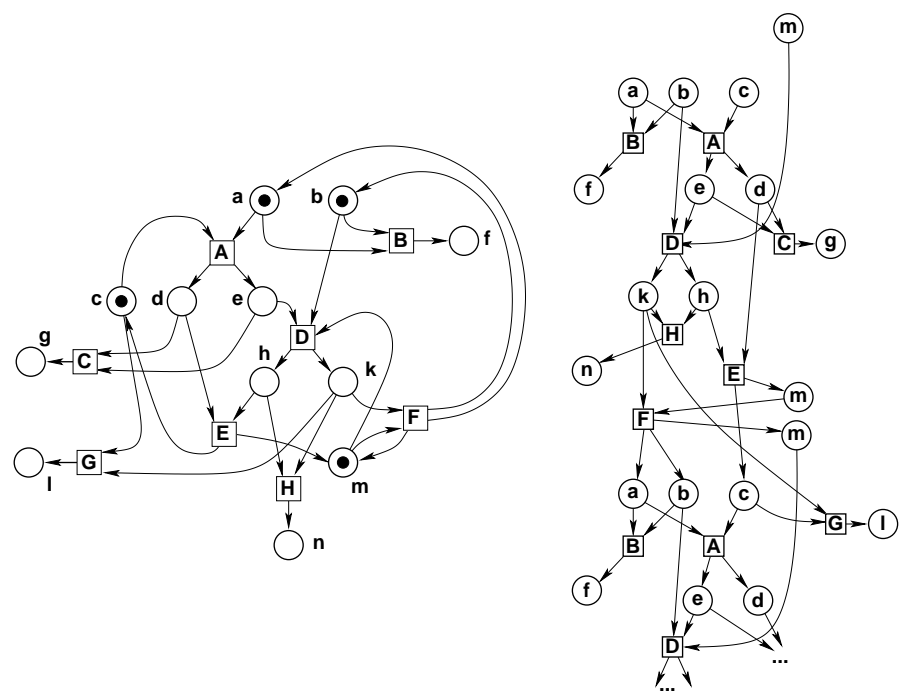

Fig. 10. A Petri net which has a choice-conformal unfolding but has infinite layers.

has a choice-conformal unfolding but still has infinite layers. Actually, this Petri net possesses only two stopping times, namely $\min \left(\Omega_{\mathcal{P}}\right)$ and the entire unfolding $\Omega_{\mathcal{P}}$. Hence choice-conformalness is not sufficient to guarantee the finiteness of layers. The very tricky topology of this counter-example suggests that "natural" Petri nets are very likely to possess finite layers.

\section{C.2 The case of free choice Petri nets}

From the above discussion, we see that we really need choice-conformalness and the finiteness of layers. This is a difficult property, not checkable directly on the Petri net, but only on the unfolding. Free choice nets are a known and simple class of Petri nets, which are choice-compact, as we will show next. Free choice nets are popular, they are regarded as an interesting intermediate class between automata and general Petri nets. For completeness, we recall the definition of free choice nets [11][5]:

Definition 5 (free choice Petri net) A Petri net $\mathcal{P}=$ $\left(P, T, \rightarrow, M_{0}\right)$ is called free choice if $\left|p^{\bullet}\right|>1$ implies that, for all $t \in p^{\bullet},|\bullet t|=1$ holds.

Lemma 4: Free choice nets are choice-compact - but the converse is not true as shown by our example of Fig. 9.

Proof: See the Appendix (in the course of this proof, we give a detailed description of the layers, for free choice nets).
C.3 Equipping choice-compact Petri nets with probabilities

For $\mathcal{P}$ a Petri net, $\Omega_{\mathcal{P}}$ its unfolding, $\omega \in \Omega_{\mathcal{P}}$, and $\mathcal{W}$ a subnet of $\Omega_{\mathcal{P}}$, define

$$
\omega_{\mathcal{W}} \triangleq \operatorname{proj}_{\mathcal{W}}(\omega)=\omega \cap \mathcal{W}
$$

the subnet obtained by removing, from $\omega$, the nodes not belonging to $\mathcal{W}$.

Any stopping time $\mathcal{B}$ is the union of all its maximal configurations. Such configurations represent partial runs $\omega_{\mathcal{B}}$. Continuing $\omega_{\mathcal{B}}$ proceeds by randomly selecting the next transition. The idea is that this choice shall be governed by the transition probability $\pi$. We formalize this next.

Consider

$$
\begin{aligned}
& L_{c} \triangleq\left\{b \in L \cap B_{c}: b^{\bullet} \subseteq L\right\} \\
& \mathcal{L}_{c} \triangleq\left\{L \in \mathcal{L}: L_{c} \neq \emptyset\right\},
\end{aligned}
$$

i.e., $\mathcal{L}_{c}$ is the set of all layers involving branching. Clearly, only layers belonging to $\mathcal{L}_{c}$ need to be randomized.

Using the generic notation (7), we identify layer $L$ with its set of "local runs" $v_{L}$, where $v \in \Omega_{\mathcal{P}}$ ranges over the set of runs which traverse $L$, i.e., satisfy $v_{L} \neq \emptyset$. Fix a prefix $\omega_{\mathcal{B}^{\prime}}$, where $L=\mathcal{B} / \mathcal{B}^{\prime}$, and write

$$
\omega_{\mathcal{B}^{\prime}} \odot v_{L}
$$

to denote that $v_{L}$ is a non-empty continuation of $\omega_{\mathcal{B}^{\prime}}$; note that we may have $v \neq \omega$.

For $L \in \mathcal{L}_{c}$, we shall now define the conditional distribution $\mathbf{P}_{L}^{\pi}\left(v_{L} \mid \omega_{\mathcal{B}^{\prime}}\right)$, for $\omega_{\mathcal{B}^{\prime}}$ fixed and $v_{L}$ ranging over the set of local runs such that:

$$
\left[v_{L} \neq \emptyset\right] \wedge\left[\omega_{\mathcal{B}^{\prime}} \odot v_{L}\right]
$$

For $v_{L}$ satisfying (9), set

$$
\mathbf{P}_{L}^{\pi}\left(v_{L} \mid \omega_{\mathcal{B}^{\prime}}\right)=\frac{1}{C_{L}} \prod_{b \in L_{c} \cap v_{L}} \pi(\varphi(e) \mid \varphi(b))
$$

where $e$ is the unique event belonging to $b^{\bullet} \cap v_{L}$, and $C_{L}$ is a normalizing constant depending on $L$ and $\omega_{\mathcal{B}^{\prime}}$, to ensure that $\mathbf{P}_{L}^{\pi}$ is a probability. Since $\mathcal{P}$ is choice-compact: $1 /$ its layers are finite and thus $L_{c}$ and $C_{L}$ are both finite, hence the product in (10) is well defined, and $2 / \varphi$ is a bijection from $b^{\bullet}$ onto $\varphi(b)^{\bullet}$, hence the term $\pi(\varphi(e) \mid \varphi(b))$ is well defined. Thus the right hand side of (10) is well defined.

We are now ready to define the restriction of our desired probability to a stopping time $\mathcal{B}$, we denote this probability by $\mathbf{P}_{\mathcal{B}}^{\pi}$. By statement 2 of lemma 3 , every stopping time $\mathcal{B}$ decomposes as a union of layers having pairwise disjoint sets of events. Set $\mathcal{L}_{\mathcal{B}} \triangleq\left\{L \in \mathcal{L}_{c}: L \subseteq \mathcal{B}\right\}$, and

$$
\mathbf{P}_{\mathcal{B}}^{\pi}\left(\omega_{\mathcal{B}}\right)=\prod_{\substack{L \in \mathcal{L}_{\mathcal{B}} \\ \omega_{L} \neq \emptyset}} \mathbf{P}_{L}^{\pi}\left(\omega_{L} \mid \omega_{\mathcal{B}^{\prime}}\right)
$$

Recall that $\omega_{\mathcal{B}^{\prime}}$ denotes the restriction of $\omega$ to $\mathcal{B}^{\prime}$, and note that it always holds that $\omega_{\mathcal{B}^{\prime}} \odot \omega_{L}$, since we consider projections of the same $\omega$, and $L$ is a continuation of $\mathcal{B}^{\prime}$. Hence 
the terms $\mathbf{P}_{L}^{\pi}\left(\omega_{L} \mid \omega_{\mathcal{B}^{\prime}}\right)$ on the right hand side of (11) are well defined. Now we claim that, if $\mathcal{B}^{\prime} \sqsubseteq \mathcal{B}$, then:

$$
\mathbf{P}_{\mathcal{B}^{\prime}}^{\pi}\left(\omega_{\mathcal{B}^{\prime}}\right)=\sum_{v: v_{\mathcal{B}^{\prime}}=\omega_{\mathcal{B}^{\prime}}} \mathbf{P}_{\mathcal{B}}^{\pi}\left(v_{\mathcal{B}}\right)
$$

Applying (12) with $\mathcal{B}^{\prime}=\min (B)$ shows in particular that formula (11) actually defines a probability, since the left hand side of (12) yields 1 in this case. Formula (12) expresses that the family $\mathbf{P}_{\mathcal{B}}^{\pi}$, where $\mathcal{B}$ ranges over the set of choice-conformal branching processes of $\mathcal{P}$, forms a projective family of probability distributions. By Kolmogorov's extension theorem [25] (see [1] for a detailed proof), there exists a unique probability $\mathbf{P}^{\pi}$ over the projective limit of the choice-conformal $\mathcal{B}$ 's, this projective limit identifies with $\Omega_{\mathcal{P}}$. This construction defines a unique probability $\mathbf{P}^{\pi}$ over $\Omega_{\mathcal{P}}$.

Hence it remains to justify (12). By induction, it is enough to show (12) for $\mathcal{B}$ and $\mathcal{B}^{\prime}$ related by $L=\mathcal{B} / \mathcal{B}^{\prime}$, for some layer $L \in \mathcal{L}_{c}$. Decompose $v_{\mathcal{B}}$ into $v_{\mathcal{B}}=\omega_{\mathcal{B}^{\prime}} \cdot v_{L}$, meaning that $v_{L}$ is a continuation of $\omega_{\mathcal{B}^{\prime}}$ and $v_{\mathcal{B}}$ is the extension of $\omega_{\mathcal{B}^{\prime}}$ by $v_{L}$. By (11) we have

$$
\omega_{\mathcal{B}^{\prime}} \odot v_{L} \Rightarrow \mathbf{P}_{\mathcal{B}}^{\pi}\left(v_{\mathcal{B}}\right)=\mathbf{P}_{\mathcal{B}^{\prime}}^{\pi}\left(\omega_{\mathcal{B}^{\prime}}\right) \times \mathbf{P}_{L}^{\pi}\left(v_{L} \mid \omega_{\mathcal{B}^{\prime}}\right),
$$

hence it remains to prove that $\sum_{v_{L}: \omega_{\mathcal{B}^{\prime}} \odot v_{L}} \mathbf{P}_{L}^{\pi}\left(v_{L} \mid \omega_{\mathcal{B}^{\prime}}\right)$ $=1$. But this results immediately from formula (10) since $\mathbf{P}_{L}^{\pi}$ is a probability. This finishes the construction of $\mathbf{P}^{\pi}$.

Some comments are in order about the present construction, versus that of [31]. The construction of [31] applies to free choice conflicts only, whereas ours is more general. It uses no Kolmogorov's extension argument, but rather performs a direct construction of the probability on a field generating the whole $\sigma$-algebra on the unfolding, and then invokes a basic extension theorem from measure theory. The resulting proof is long, like ours, and it is more technical. Our proof uses our notion of stopping time, which turns out to be required anyway for the Markov property we investigate next - the latter topic is not considered in $[30][31]$.

Running example, continued. Let us provide explicit formulas for our running example of Fig. 9. Referring to this figure, set $\mathcal{B}_{0}=\min \left(\Omega_{\mathcal{P}}\right)$, and denote by $L_{1}$ and $L_{2}$ the two layers in light and mid grey, respectively, and let $\mathcal{B}_{1}=L_{1}$ and $\mathcal{B}_{2}=\mathcal{B}_{1} \cup L_{2}$ be the two corresponding stopping times. The two layers $L_{1}$ and $L_{2}$ contain local runs, we denote them by $w_{L_{i}}^{j}$. We list them here as unions of graphs using the labels from Fig. 9:

$$
\begin{aligned}
& \varphi\left(w_{L_{1}}^{1}\right)=(7) \bigcup(1) \rightarrow i i \rightarrow(2) \rightarrow i i i \rightarrow(1) \\
& \varphi\left(w_{L_{1}}^{2}\right)=(1,7) \rightarrow i \rightarrow(2,3) \bigcup(2) \rightarrow i i i \rightarrow(1) \\
& \varphi\left(w_{L_{2}}^{1}\right)=(3,4) \rightarrow i v \rightarrow(7,5) \bigcup(4) \rightarrow v i \rightarrow(5) \\
& \varphi\left(w_{L_{2}}^{2}\right)=(4) \rightarrow v \rightarrow(6)
\end{aligned}
$$

Layer $L_{1}$ can continue any co-set labeled by 1,7 ; thus referring to formula (10), we have:

$$
\left.\begin{array}{l}
\mathbf{P}_{L_{1}}^{\pi}\left(w_{L_{1}}^{1} \mid \omega_{\mathcal{B}_{0}}=\omega_{0}\right)=\pi(i i \mid 1) \\
\mathbf{P}_{L_{1}}^{\pi}\left(w_{L_{1}}^{2} \mid \omega_{\mathcal{B}_{0}}=\omega_{0}\right)=\pi(i \mid 1)
\end{array}\right\}
$$

where $\omega_{0}=\min \left(\Omega_{\mathcal{B}}\right)$. Since all runs begin with $\omega_{0}$, the conditioning in (13) is trivial and could be omitted. Layer $L_{2}$ is a continuation of $\mathcal{B}_{1}=L_{1}$. For runs beginning with $\omega_{\mathcal{B}_{1}}=w_{L_{1}}^{1}$ as a prefix, the only possible continuation is $w_{L_{2}}^{2}$, this justifies formula (14). For runs beginning with $\omega_{\mathcal{B}_{1}}=w_{L_{1}}^{2}$ as a prefix, two extensions, by $w_{L_{2}}^{j}, j=1,2$ are possible, and formula (15) follows.

The possible prefixes within $\mathcal{B}_{2}$ are given by $\omega_{\mathcal{B}_{2}}^{i, j} \triangleq w_{L_{1}}^{i} \cup$ $w_{L_{2}}^{j}$, for $i, j=1,2$, and we have - note that $\mathbf{P}_{\mathcal{B}_{2}}^{\pi}$ is indeed a probability:

$$
\begin{aligned}
\mathbf{P}_{\mathcal{B}_{2}}^{\pi}\left(\omega_{\mathcal{B}_{2}}^{1,1}\right)=\pi(i i \mid 1) \times 0 & \mathbf{P}_{\mathcal{B}_{2}}^{\pi}\left(\omega_{\mathcal{B}_{2}}^{1,2}\right)=\pi(i i \mid 1) \times 1 \\
\mathbf{P}_{\mathcal{B}_{2}}^{\pi}\left(\omega_{\mathcal{B}_{2}}^{2,1}\right)=\pi(i \mid 1) \times \pi(i v \mid 4) & \mathbf{P}_{\mathcal{B}_{2}}^{\pi}\left(\omega_{\mathcal{B}_{2}}^{2,2}\right)=\pi(i \mid 1) \times \pi(v \mid 4)
\end{aligned}
$$

Discarding the prefixes having zero probability, we find three possible prefixes, with respective probabilities $\pi(i i \mid 1), \pi(i \mid 1) \times \pi(i v \mid 4), \pi(i \mid 1) \times \pi(v \mid 4)$. We insist that this is different from randomizing firing sequences, we rather randomize "firing sequences up to interleavings".

\section{The Markov property}

We first define the $\sigma$-algebras of past, present, and future. Using notation (7), we consider the following equivalence relation on $\Omega_{\mathcal{P}}$ :

$$
\begin{aligned}
& \omega \sim \mathcal{W} \omega^{\prime} \text { iff } \\
& \omega_{\mathcal{W}} \text { and } \omega_{\mathcal{W}}^{\prime} \text { are isomorphic, } \\
& \text { when seen as labelled graphs. }
\end{aligned}
$$

Note that definition (16) for the relation $\sim_{\mathcal{W}}$ simplifies when $\mathcal{W}$ is a branching process, since isomorphism reduces to equality in this case. Then we define the $\sigma$-algebra $\mathcal{F}_{\mathcal{W}}$ as follows:

$$
\left.A \in \mathcal{F}_{\mathcal{W}} \quad \text { iff } \quad \begin{array}{c}
\omega \in A \\
\omega^{\prime} \sim \mathcal{W} \omega
\end{array}\right\} \Rightarrow \omega^{\prime} \in A .
$$

Consider $\mathcal{B}$ a stopping time, and denote by $\mathcal{B}_{+}$the suffix of $\Omega_{\mathcal{P}}$ equal to:

$$
\mathcal{B}_{+}=\left(\Omega_{\mathcal{P}} \backslash \mathcal{B}\right) \cup \cdot\left(\Omega_{\mathcal{P}} \backslash \mathcal{B}\right),
$$

where $\cup$ denotes the union of labelled graphs. $\mathcal{B}_{+}$is to be interpreted as the future of $\mathcal{B}$. Then we set

$$
X_{\mathcal{B}}=\mathcal{B} \cap \mathcal{B}_{+},
$$

and we call it the present of $\mathcal{B}$. Accordingly, the $\sigma$-algebras of past, present, future are, respectively:

$$
\mathcal{F}_{\mathcal{B}}, \quad \mathcal{X}_{\mathcal{B}} \triangleq \mathcal{F}_{X_{\mathcal{B}}} \quad \mathcal{F}_{\mathcal{B}}^{+} \triangleq \mathcal{F}_{\mathcal{B}_{+}},
$$


where the generic definition (17) was used. Note that the present $\mathcal{X}_{\mathcal{B}}$ consists of sets of conditions that are either mutually concurrent or in conflict, but not causally related.

The example of Fig. 11 is an interesting illustration of

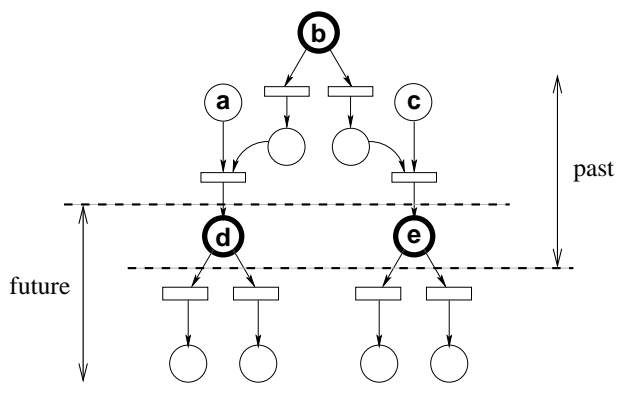

Fig. 11. Past, present, and future.

our Markov property. In this figure, we show an unfolding. The "past" area depicts a stopping time, we call it $\mathcal{B}$. The "future" area depicts $\mathcal{B}_{+}$. Hence the present $X_{\mathcal{B}}$ consists of the two conditions d,e. Now, the exit cut containing d (resp. e) is $\{c, d\}$ (resp. $\{a, e\}$ ) - by exit cut, we mean the cut by which the configuration containing $d$ (resp. e) exits $\mathcal{B}$. Thus the present $X_{\mathcal{B}}$ is smaller than the set of exit cuts of $\mathcal{B}$ it defines. In fact, conditions $a, b$ belonging to these cuts are useless for predicting the future! This illustrates once again that our model takes deep advantage of the underlying concurrency. We are now ready to state our theorem.

Theorem 1 (strong Markov property) Future and past are conditionally independent, given the present:

$$
\forall B \in \mathcal{F}_{\mathcal{B}}^{+}: \mathbf{P}^{\pi}\left(B \mid \mathcal{F}_{\mathcal{B}}\right)=\mathbf{P}^{\pi}\left(B \mid \mathcal{X}_{\mathcal{B}}\right) .
$$

Since all $\mathcal{B}$ we consider are stopping times, formula (21) is in fact a strong Markov property.

Proof: See the Appendix.

\section{E. In Markov nets, concurrency matches stochastic inde-} pendence

Theorem 2 (conditional independence of layers) Consider the partial order $(\mathcal{L}, \prec)$ introduced in lemma 3 , and let $L_{1} \neq L_{2}$ be two layers such that neither $L_{1} \prec L_{2}$ nor $L_{2} \prec L_{1}$ holds. Denote by $\mathcal{B}$ the minimal stopping time such that $L_{i}$ is a continuation of $\mathcal{B}$ for $i=1,2$ (such a $\mathcal{B}$ exists). Then the two $\sigma$-algebras $\mathcal{F}_{L_{1}}$ and $\mathcal{F}_{L_{2}}$ are conditionally independent given $\mathcal{F}_{\mathcal{B}}$, meaning that, for $A_{i} \in \mathcal{F}_{L_{i}}, i=1,2$, we have

$$
\begin{aligned}
& \mathbf{P}^{\pi}\left(A_{1} \cap A_{2} \mid \mathcal{F}_{\mathcal{B}}\right)=\mathbf{P}^{\pi}\left(A_{1} \mid \mathcal{F}_{\mathcal{B}}\right) \times \mathbf{P}^{\pi}\left(A_{2} \mid \mathcal{F}_{\mathcal{B}}\right) \\
& \quad \text { Proof: See [1]. }
\end{aligned}
$$

Note that, if $\mathcal{P}$ is an automaton, then (22) boils down to $0=0$ and our theorem is trivial (this is why no such result was stated for stochastic automata). Now, if layers $L_{1}$ and $L_{2}$ are concurrent, then $A_{1} \cap A_{2} \neq \emptyset$ and the theorem is non trivial. It expresses that concurrent continuations are conditionally independent, given the past.
Running example, continued. Our running example of Fig. 9 is too small to illustrate theorem 2, because it does not exhibit enough concurrency. However, if we apply theorem 2 to Fig. 6-bottom, we derive that the two noninteracting parts of $\mathcal{P}$ are probabilistically independent. This was our second requirement, when discussing Fig. 6.

\section{HANdLing General SAFE NETS}

This section is devoted to the analysis of Petri nets which are not choice-compact. We will show how to make such nets free choice, and hence choice-compact. In Fig. 12 we

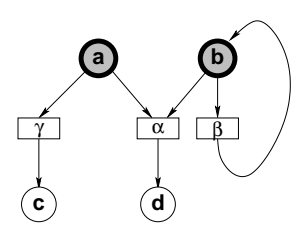

(a)

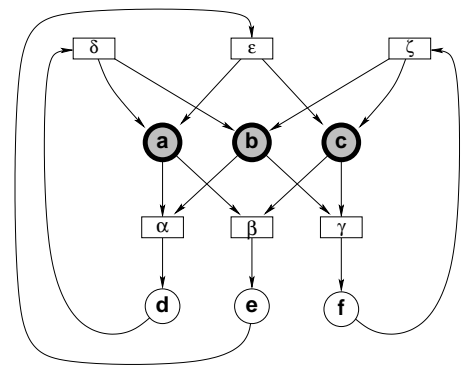

(b)
Fig. 12. Two examples that are not free choice.

show two Petri nets, for further discussion in the sequel. None of them is choice-compact, but we still wish to investigate how they can be randomized.

\section{A. Using pre-selection}

Pre-selection has been proposed as a way to transform a net exhibiting constrained choice into another net which is free choice. It consists in breaking the output branches of a place exhibiting constrained choice, by inserting, on each branch, a pair $\{$ transition $\rightarrow$ place $\}$. Pre-selection separates blocking from synchronization. We illustrate the use of this technique on the Petri nets of the Fig. 12, the result is shown in Fig. 13. The nets of Fig. 12 (a) and (b), (a)

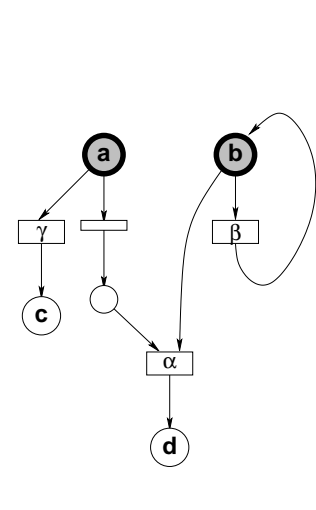

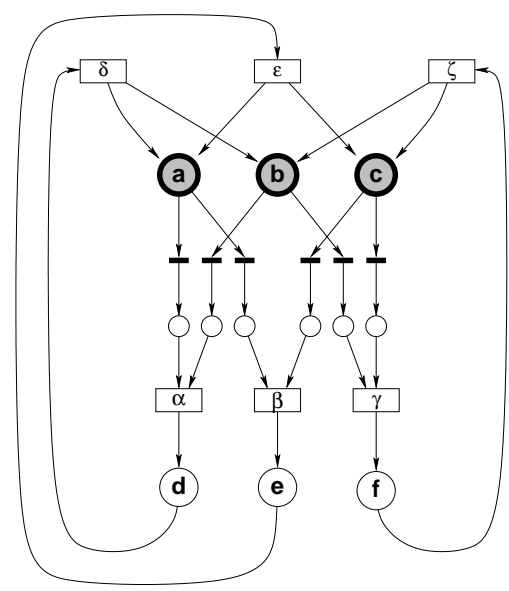

(b)
Fig. 13. Making the nets of Fig. 12 free choice, using preselection.

are modified, in Fig. 13 (a) and (b) respectively, by enforcing preselection: dummy transitions and places are added, 
they are figured by small boxes and circles. We formalize this construction next.

Pre-selection. We are given a net $\mathcal{P}$ with its set $P_{c}$ of places exhibiting choice, and let $\Omega_{\mathcal{P}}=(B, E, \rightarrow, \varphi)$ be its unfolding. Consider the following relation $\mathcal{C}_{c} \subseteq(B \times E) \times(P \times T)$ :

$$
((b, e),(p, t)) \in \mathcal{C}_{c} \quad \text { iff }\left\{\begin{array}{cl}
p \in P_{c} & \\
e \in b^{\bullet} & , \quad t \in p^{\bullet} \\
\varphi(b)=p & , \quad \varphi(e)=t
\end{array}\right.
$$

Fix $b, p, t$, and suppose that:

the set $\left\{e \mid((b, e),(p, t)) \in \mathcal{C}_{c}\right\}$ has cardinal $>1$.

Then,

replace the branch $p \rightarrow t$, in the flow relation of $\mathcal{P}$, by the path $p \rightarrow t_{p, t} \rightarrow p_{p, t} \rightarrow t$,

where $\left(t_{p, t}, p_{p, t}\right)$ is a pair of new (dummy) transition and place which we add to the original net $\mathcal{P}$. In other words, if some condition labelled by a branching place $p$ has at least two different events in its postset that are labelled by the same transition, then we add a pair of dummy transition and place to each outgoing branch of place $p$ in net $\mathcal{P}$. Note that this new dummy place exhibits no choice, and therefore we do not need to modify the transition probability $\pi(t \mid p)$. Performing this for each case in which (24) holds, we construct a free choice net $\widehat{\mathcal{P}}$, for which condition $(24)$ never holds. Applying to $\widehat{\mathcal{P}}$ the theory of Section III-C, we get an extended probability space

$$
\left\{\widehat{\Omega}_{\mathcal{P}}, \widehat{\mathcal{F}}, \widehat{\mathbf{P}}^{\pi}\right\}
$$

where $\pi$ is as before, we call it the extended Markov net ${ }^{2}$ associated with $\mathcal{P}$. Of course, a natural question is: how to take advantage of this extended Markov net in defining a probabilistic version of the original net $\mathcal{P}$ ? We investigate this next.

Relation between the extended Markov net, and the original net. One may guess that erasing, in the extended unfolding $\widehat{\Omega}_{\mathcal{P}}$, dummy conditions and events, would yield again $\Omega_{\mathcal{P}}$. This may not be the case, however. In fact, since preselection has been performed, there are in general maximal configurations belonging to $\widehat{\Omega}_{\mathcal{P}}$, which terminate at dummy conditions. Erasing dummy conditions and events in such a configuration may yield either a maximal configuration of $\Omega_{\mathcal{P}}$, or a prefix of a maximal configuration of $\Omega_{\mathcal{P}}$. This means that erasing, in the extended unfolding $\widehat{\Omega}_{\mathcal{P}}$, dummy conditions and events, yields in general an additional set of partial runs. Hence the so obtained set is larger than $\Omega_{\mathcal{P}}$, seen as a set of runs. We shall investigate this situation later, but we first discuss the case in which such an artifact does not occur.

Consider the extended Petri net $\widehat{\mathcal{P}}$, its unfolding $\widehat{\Omega}_{\mathcal{P}}$ possesses "dummy" paths, themselves labelled by paths of the

\footnotetext{
2 The term "extended" used here should not be confused with its use in "extended free choice".
}

form (25). Denote these paths by $b_{p} \rightarrow e_{p, t} \rightarrow b_{p, t}$, where $\varphi\left(b_{p}\right)=p, \varphi\left(e_{t}\right)=t$. Assume for the moment that:

Assumption 1: Replacing, in $\widehat{\Omega}_{\mathcal{P}}$, the path $b_{p} \rightarrow e_{p, t} \rightarrow$ $b_{p, t}$ by the single node $b_{p}$, defines a map $\Psi: \widehat{\Omega}_{\mathcal{P}} \mapsto$ $\Omega_{\mathcal{P}}$, (where $\widehat{\Omega}_{\mathcal{P}}$ and $\Omega_{\mathcal{P}}$ are seen as sets of runs) which is measurable, from $\widehat{\mathcal{F}}$ to $\mathcal{F}$.

Note that in this case, the map $\boldsymbol{\Psi}$ may or may not be one-to-one, but, clearly, it is onto. Then

$$
\left\{\Omega_{\mathcal{P}}, \mathcal{F}, \mathbf{P}^{\pi}\right\}, \text { where } \quad \mathbf{P}^{\pi}=\Psi^{-1}\left(\widehat{\mathbf{P}}^{\pi}\right),
$$

is a probability space, we can define it as the Markov net generated by $\pi$ on $\mathcal{P}$. A characterization of the safe nets $\mathcal{P}$ for which assumption 1 holds true is given in Lemma 5 below.

An example and a counter-example of Petri net satisfying assumption 1 are shown in Fig. 14, (a) and (b), respectively. In this figure, we show the unfoldings of the nets shown in

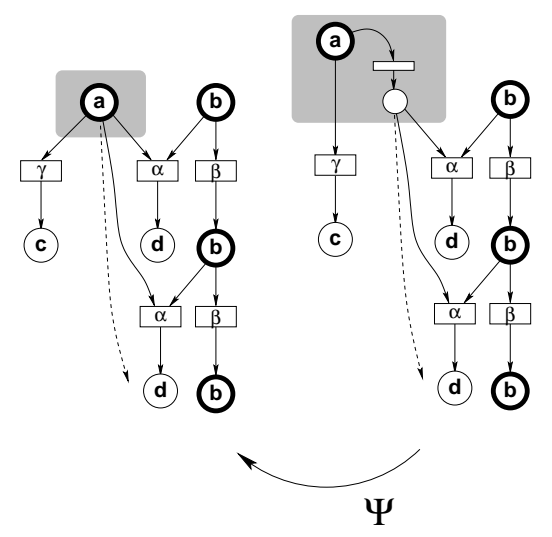

(a)

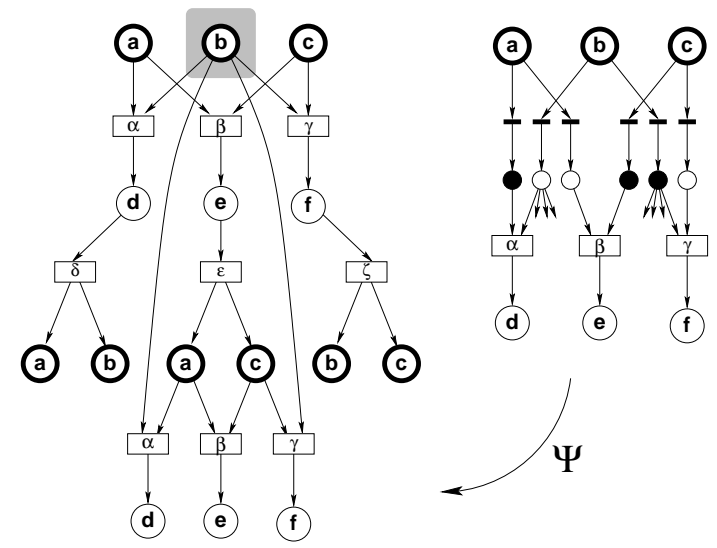

(b)

Fig. 14. Showing the unfoldings of the nets of figures $12 / 13$, (a) and (b), respectively. Unfolding (a) satisfies assumption 1, whereas unfolding (b) does not.

Fig. 13, (a) and (b), respectively. It turns out that $\boldsymbol{\Psi}$ is a one-to-one map from $\widehat{\Omega}_{\mathcal{P}}$ to $\Omega_{\mathcal{P}}$, for the unfolding (a). However, $\boldsymbol{\Psi}$ does not result in a map from $\widehat{\Omega}_{\mathcal{P}}$ to $\Omega_{\mathcal{P}}$, for the unfolding (b). Indeed, after applying pre-selection for 
example (b), the three conditions in black in the unfolding constitute a maximal coset, and therefore constitute the termination of some finite configuration. This configuration is blocked at dummy, additional conditions, and therefore $\boldsymbol{\Psi}$ maps it to a configuration which is the strict prefix of some run of the original net. This strict prefix is not a run, hence $\boldsymbol{\Psi}$ does not define a map from $\widehat{\Omega}_{\mathcal{P}}$ to $\Omega_{\mathcal{P}}$. We formalize this next.

\section{B. How to check if pre-selection fails to work: doubly com- plete prefix}

In this subsection we analyze how to check assumption 1. This is not obvious, as this assumption is formulated in terms of the unfoldings, which are infinite objects. Our objective is to provide an effective criterion to check this assumption. The following lemma is useful, as a first step toward achieving this objective:

Lemma 5: Assumption 1 is violated iff there exists $\widehat{\omega} \in$ $\widehat{\Omega}_{\mathcal{P}}$, satisfying the following conditions:

(i) some maximal node of $\widehat{\omega}$ is a dummy condition, and

(ii) if $\omega_{d}$ denotes the configuration obtained by erasing, in $\widehat{\omega}$, the maximal dummy conditions and corresponding dummy events, then $\omega_{d}$ possesses another extension $\widehat{\omega}^{\prime} \in$ $\widehat{\Omega}_{\mathcal{P}}$ with no maximal dummy conditions.

Proof: We first prove the "if" part. Due to (ii), removing the dummy conditions and events from maximal configuration $\widehat{\omega}^{\prime}$ yields a maximal configuration $\omega^{\prime} \in \Omega_{\mathcal{P}}$. On the other hand, performing the same for $\widehat{\omega}$ yields a strict sub-configuration of the same $\omega^{\prime}$,

$$
\text { we call it } \omega_{d} \text {. }
$$

Hence $\omega_{d}$ is not a maximal configuration of the unfolding of $\mathcal{P}$, and is therefore not an element of $\Omega_{\mathcal{P}}$. In fact, our operation maps elements of the extended net to not necessarily maximal configurations of the original net. Hence assumption 1 is violated.

To prove the "only if" part, we proceed by contradiction. Assume first that condition (i) is not satisfied. Then clearly assumption 1 holds true - in this case, the map $\boldsymbol{\Psi}$ is oneto-one. Next, assume that (i) is satisfied, but (ii) is not. Then all proper extensions of $\omega_{d}$ possess maximal dummy places. Hence all such maximal configurations of $\widehat{\Omega}_{\mathcal{P}}$ are mapped to $\omega_{d}$, thus $\omega_{d}$ is a maximal configuration of $\Omega_{\mathcal{P}}$. Again, assumption 1 holds true in this case.

Complete prefixes, and doubly complete prefixes. We are still not happy, since lemma 5 remains formulated in terms of unfoldings and runs. We shall use the concept of complete prefix and its new related notion we propose here, we call it the doubly complete prefix. McMillan [24] and, later, Esparza and Römer [14], have shown that reachability properties of Petri net unfoldings are revealed by some finite prefixes of them, called complete:

Definition 6 (finite complete prefix) We say that a branching process $\mathcal{B}$ of a Petri net $\mathcal{P}$ is complete if, for every reachable marking $M$ of $\mathcal{P}$, and for every transition $t$ enabled by $M$, there exists a cut $\mathbf{c}$ of $\mathcal{B}$ labelled by $M$ (i.e., $M$ is represented in $\mathcal{B})$, and there exists an event $e \in \mathbf{c}^{\bullet}$ which is labelled by $t$ (i.e., $t$ is represented by $e$ ).
It has been proved by McMillan [24] that finite complete prefixes exist for every Petri net unfolding, and Esparza and Römer [14] have provided an efficient algorithm for computing a complete prefix, which is minimal in some sense detailed in this reference. This minimal complete prefix of Petri net $\mathcal{P}$ is denoted by $\mathcal{B}_{\mathcal{P}}$.

Unfortunately, complete prefixes are not enough in general to check Assumption 1, or, equivalently, the two conditions of Lemma 5. We need to consider the doubly complete prefix, which is described informally as follows. Take the complete prefix, and make enough copies of it. The initial marking is represented by the minimal cut of the prefix, and also by some other cuts, lying at the "exit boundary" of the prefix. Re-paste a copy of the complete prefix at each "exit" cut representing the initial marking, this yields the doubly complete prefix.

This construction is illustrated in Fig. 15. In this fig-
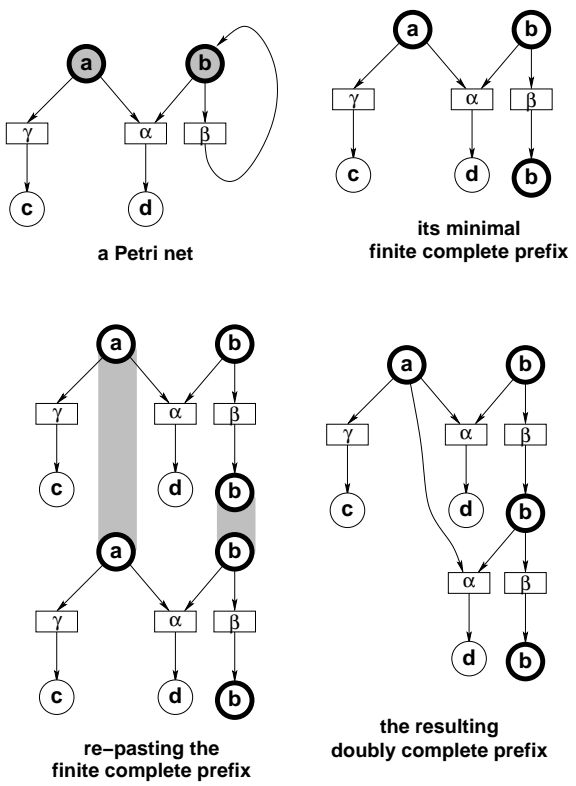

Fig. 15. Doubly complete prefix. Note that only one instance of the branch $\mathrm{a} \rightarrow \gamma \rightarrow \mathrm{c}$ is kept after re-pasting.

ure, we show a Petri net (top-left) and its complete prefix (top-right). In this complete prefix, there are two cuts associated with the marking a,b: the minimal cut, and one of the exit cuts. In the bottom-left diagram, we show two copies of the complete prefix. The minimal cut of the second copy is superimposed on the exit cut of the first copy, this is depicted by the two grey thick links. Then, the two branches $\mathrm{a} \rightarrow \gamma \rightarrow \mathrm{c}$ are also superimposed, this yields the doubly complete prefix shown in the bottom-right diagram.

Let us formalize this construction. Let $\mathcal{P}$ be a Petri net and let $\mathcal{B}_{\mathcal{P}}$ a minimal complete prefix. In what follows we write $\mathcal{B}$ instead of $\mathcal{B}_{\mathcal{P}}$ for the sake of clarity. For $M$ a - reachable marking of $\mathcal{P}$, we denote by $\mathcal{P}_{M}$ the Petri net obtained by substituting $M$ for the initial marking $M_{0}$ in $\mathcal{P}: \mathcal{P}_{M}=(P, T, \rightarrow, M)$; denote by $\mathcal{B}_{M}$ a minimal complete prefix of $\mathcal{P}_{M}$ (hence $\mathcal{B}=\mathcal{B}_{M_{0}}$ ), and denote by $\varphi_{M}$ the homomorphism defining the unfolding of Petri net $\mathcal{P}_{M}$. For each reachable marking $M$ of $\mathcal{P}$, denote by $\mathbf{C}_{M}$ the set of 
the cuts contained in $\mathcal{B}$ which represent $M, \mathbf{C}_{M}$ is non empty since $\mathcal{B}$ is complete. For each $\mathbf{c} \in \mathbf{C}_{M}$, the following formulas define an injective homomorphism $\psi_{\mathbf{c}}: \mathcal{B}_{M} \mapsto$ $\Omega_{\mathcal{P}}:$

$$
\begin{array}{ccc}
\psi_{\mathbf{c}}\left(\min \left(\mathcal{B}_{M}\right)\right)=\mathbf{c} & , \quad \varphi\left(\psi_{\mathbf{c}}(n)\right)=\varphi_{M}(n), \\
\psi_{\mathbf{c}}(\bullet)=\bullet\left(\psi_{\mathbf{c}}(e)\right) & , \quad \psi_{\mathbf{c}}\left(e^{\bullet}\right)=\left(\psi_{\mathbf{c}}(e)\right)^{\bullet}
\end{array}
$$

Finally, we set

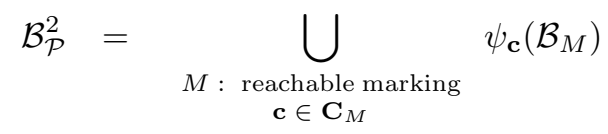

Definition 7 (doubly complete prefix) Formulas $(29,30)$ yield a branching process of $\mathcal{P}$ which is an extension of the complete prefix $\mathcal{B}_{\mathcal{P}}$, we call it the doubly complete prefix of $\mathcal{P}$ and denote it by $\mathcal{B}_{\mathcal{P}}^{2}$. It satisfies the following property: for each reachable marking $M$, and each marking $M^{\prime}$ reachable from $M, \mathcal{B}_{\mathcal{P}}^{2}$ contains a configuration reaching a cut representing $M$, and terminating at a cut representing $M^{\prime}$.

Using this notion we can state the following theorem:

Theorem 3: The conditions of Lemma 5 can be checked on the doubly complete prefix of Petri net $\widehat{\mathcal{P}}$.

Proof: See the Appendix.

$\diamond$

\section{What happens if pre-selection fails to work}

From the proof of Lemma 5 it can be deduced that the action of removing the dummy places and transitions from the maximal configurations $\widehat{\omega} \in \widehat{\Omega}_{\mathcal{P}}$, can be made a map

$$
\Psi \quad: \quad \widehat{\Omega}_{\mathcal{P}} \longmapsto\left(\Omega_{\mathcal{P}} \cup \Omega_{\mathcal{P}}^{\text {block }}\right)
$$

where $\Omega_{\mathcal{P}}^{\text {block }}$ denotes the set of (non maximal) configurations $\omega_{d}$ of $\Omega_{\mathcal{P}}$ obtained as in (28). This map $\boldsymbol{\Psi}$ is oneto-one, from the subset of configurations which do not terminate at dummy places, onto $\Omega_{\mathcal{P}}$; and it is only onto, from the subset of configurations which terminate at some dummy place, onto $\Omega_{\mathcal{P}}^{\text {block }}$. Therefore $\Omega_{\mathcal{P}}^{\text {block }}$ models the "blocking" of the configurations subject to a deadlocked choice of the routers as in Theorem 3.

Equip $\left(\Omega_{\mathcal{P}} \cup \Omega_{\mathcal{P}}^{\text {block }}\right)$ with its natural $\sigma$-algebra, we denote it again by $\mathcal{F}$. Then we can again proceed as in $(27)$ and get a probability space

$$
\left\{\left(\Omega_{\mathcal{P}} \cup \Omega_{\mathcal{P}}^{\text {block }}\right), \mathcal{F}, \mathbf{P}^{\pi}\right\} \quad, \quad \mathbf{P}^{\pi}=\Psi^{-1}\left(\widehat{\mathbf{P}}^{\pi}\right),
$$

in which blocking configurations are made explicit. Of course, due to the additional set $\Omega_{\mathcal{P}}^{\text {block }}$, the original unfolding $\Omega_{\mathcal{P}}$ suffers from a "loss of mass", i.e., we expect that $\mathbf{P}^{\pi}\left(\Omega_{\mathcal{P}}\right)<1$. In fact the following zero/one law holds:

Theorem 4: Consider a net $\mathcal{P}$ satisfying conditions (i,ii) of lemma 5 , we use the notations of this lemma. Denote by $c$ the set of maximal conditions of $\widehat{\omega} \cap \widehat{\omega^{\prime}}$, i.e., the co-set at which $\widehat{\omega}$ and $\widehat{\omega^{\prime}}$ branch from each other. Assume that the sub-marking $\varphi(c)$ is reachable from any initial marking. Then we have $\mathbf{P}^{\pi}\left(\Omega_{\mathcal{P}}\right)=0$.
This theorem means that infinite behaviours of the original Petri net have a zero probability with respect to probability distribution $\mathbf{P}^{\pi}$. Of course, finite behaviours have a non zero probability, so distribution $\mathbf{P}^{\pi}$ is still of interest at describing the probabilistic behaviour of finite prefixes of runs.

Proof: See the Appendix.

$\diamond$

\section{RELATED WORK}

The problem of distributed diagnosis of discrete event systems motivated the present fundamental work, we refer the reader to [12] and the references therein. On the other hand, to our knowledge, net unfoldings are almost unkown in the control community. The only references we are aware of are [22][23], both in the context of supervisory control of discrete event systems modelled by Petri nets.

Having random variables indexed by sets that are not linearly ordered has already been considered in the litterature, in the area of probability on processes indexed by finite graphs. This topic has its origin in the pioneering work of A.P. Dempster in the late seventies, when the so-called Dempster-Shafer theory of belief functions and belief networks was introduced in statistics and artificial intelligence (see Shafer [29]). Since then, this topic has known significant developments, but to our knowledge no generalization to interacting stochastic processes (i.e., with dynamics and infinite behaviours) has been developed. Benveniste, Lévy and Fabre have proposed more recently [7] a theory of interacting finite systems of random variables, but again no generalization to stochastic processes with dynamics was available. In [2] Fabre et al. have considered for the first time a new class of stochastic Petri nets in which interleaving of concurrent transitions was not randomized; they were called Partially Stochastic Petri nets (PSPN). In [2] an in-depth comparison of this new model with existing stochastic generalizations of Petri nets (mostly for performance evaluation purposes) is provided. Our purpose in [2] was somewhat limited, although more concrete: Also motivated by distributed diagnosis of telecommunications networks, we proposed a generalization of the Viterbi algorithm for maximum likelihood estimation of the hidden state trajectory from a sequence of observations. See [16] for a detailed description of the resulting distributed algorithms. Since only finite runs were considered, this needed only a limited understanding of the underlying theory, and the full underlying probability for such PSPN was not constructed; the present paper provides a full development of such a theory, under the name of Markov nets. Related work on combining Petri nets with probabilities was already discussed in section II-A.

The work closest to ours is due to Hagen Völzer [30]. Besides ours, this work is the only one we know in the context of the standard unfolding semantics, in which partial orders, not interleavings, are randomized. This interesting work was motivated by a different application, namely the modeling of distributed algorithms equipped with a coin flip construct. We refer the reader to [30] for the discussion of the related bibliography. In fact, Hagen Völzer proposes 
a construction equivalent to ours, for the case of free choice conflicts, but with a different proof of its construction (the proof is available in [31]). He does not consider stopping times and Markov properties, and does not study the extension to general safe Petri nets. On the other hand, he focuses on the comparison of sequential versus concurrent semantics, and fairness.

Finally, motivated by the findings of the present work, S. Haar has proposed [20] an approach to probabilizing partial order behaviors, in which the unfolding semantics is modified. In fact, rather than the token/place-oriented view of the branching process semantics, his approach is based on a cluster view (with clusters being subnets as defined in the proof of lemma 2). Each net being disjointly partitioned into its clusters, probabilistic choice of actions can be made within each cluster; the events of the resulting parallel runs correspond to firings of multi-sets of transitions, rather than single transitions as in the present work. The probability of the firing events can thus be renormalized on a finite static substructure of the net, rather than on layers of the branching process unfoldings (recall that these layers can grow infinitely long). As a result, the cluster approach is always applicable without restrictions to arbitrary Petri nets. The cost is, of course, the use of a different semantics, with an additional effort necessary to include the scheduling of clusters; see the application, in [19], of this to partial order fairness. Both branching process and cluster approaches have their respective merits and drawbacks, and should be seen as complementing one another.

\section{Discussion AND PERSPECTIVES}

We have proposed a new theory for extending Markov chains, semi-Markov chains, or HMM's to distributed networked systems. Our generalization was called Markov nets, to account for its tight relation to Petri nets. Our model offers means for handling local state and time, and concurrency. Markov nets can be composed, and in doing so, non interacting components are independent in the probabilistic sense, and more generally, independence matches concurrency. Runs of Markov nets are partial orders, and therefore are insensitive to interleavings. To our knowledge, no previously existing probabilistic model for communicating automata or Petri nets has these features.

We have used unfoldings of Nielsen, Plotkin, and Winskel [26], see also [13] and [14], [15]. We have considered here the case of unfoldings for 1-safe nets, but the reader should remember that the unfolding technique can be extended to general nets, see [18][17]. While preparing this work, we were not expecting that looking for an adequate concept of time and associated $\sigma$-algebras, we would discover stopping times and layers, two concepts that seem to be of interest per se in Petri net unfolding theory.

This research effort was motivated by the case of distributed diagnosis and event correlation in telecommunications networks and services, see [16]. In the latter paper, distributed diagnosis is regarded as the problem of estimating the most likely hidden state trajectory from dis- tributed observations, by performing a new kind of "distributed Viterbi" algorithm.

Besides performing hidden state estimation via distributed Viterbi algorithm, our study allows also to envision the learning of the parameters of this stochastic system, from distributed observations. Our next objective is to generalize existing EM-type of algorithms used for НMм identification, to the distributed case. Also, one can imagine that this framework opens the route toward probabilistic simulation and performance evaluation tools for large distributed networked systems.

\section{APPENDIX}

\section{Proof of Lemma 1}

Assume first that $\mathcal{B}=\mathcal{B}_{\tau}$ for some stopping time $\tau$. Select some condition $b \in \mathcal{B}_{\tau}$ such that $\varphi(b) \in P_{c}$ and $b^{\bullet} \cap \mathcal{B}_{\tau} \neq \emptyset$. Denote by $n$ the number of steps needed to reach $b$. Since $b^{\bullet} \cap \mathcal{B}_{\tau} \neq \emptyset$, there exists a run $\omega$ containing $b$ and such that $\tau(\omega)>n$. Since $\tau$ is a stopping time, for any $\omega^{\prime}$ such that $\omega_{n}^{\prime}=\omega_{n}$, we also have $\tau\left(\omega^{\prime}\right)>n$. Hence $b^{\bullet} \cap \omega^{\prime} \subseteq \mathcal{B}_{\tau}$, this proves the only if part.

To prove the if part, assume that $\mathcal{B}$ satisfies the property stated in the lemma. For every run $\omega$, define $\tau(\omega)$ as being the length of the prefix $\omega \cap \mathcal{B}$. Pick a run $\omega$ and set $n=$ $\tau(\omega)$. Select an arbitrary $\omega^{\prime}$ such that $\omega_{n}^{\prime}=\omega_{n}$. The following cases need to be considered. 1/ $\omega_{n+1}^{\prime}=\omega_{n+1}$ : then, $\tau\left(\omega^{\prime}\right)=\tau(\omega) .2 / \omega_{n+1}^{\prime} \neq \omega_{n+1}$ but $\omega_{n+1}^{\prime} \not \subset \mathcal{B}$ : then, again $\tau\left(\omega^{\prime}\right)=\tau(\omega) .3 / \omega_{n+1}^{\prime} \neq \omega_{n+1}$ but $\omega_{n+1}^{\prime} \subseteq \mathcal{B}$ : the latter case cannot occur, since it violates the special assumption on $\mathcal{B}$. Thus, $n=\tau(\omega)$ and $\omega_{n}^{\prime}=\omega_{n}$ together imply $\tau\left(\omega^{\prime}\right)=\tau(\omega)$, hence $\tau$ is a stopping time.

\section{Proof of Lemma 4}

Lemma 4 is an immediate corollary of the following lemma, which characterizes the structure of layers for free choice nets (see (5) for the definition of $B_{c}$ ):

Lemma 6: Let $L$ be a layer of $\Omega_{\mathcal{P}}$, and denote by ${ }^{\circ} L \triangleq L \cap \bullet L$ the "interior" of $L$. Then $\left|{ }^{\circ} L \cap B_{c}\right| \leq 1$. Denote by $b_{L}$ the unique branching condition of ${ }^{\circ} L$, if any. Then: $b_{L} \in \min (L)$. Also, $\varphi$ is a bijection from $b_{L}^{\bullet}$ onto $\varphi\left(b_{L}\right)^{\bullet}$.

Proof: The last statement is easy, so we focus on the other ones. Consider the canonical decomposition of $L$, namely $L=\mathcal{B}^{\prime \prime} / \mathcal{B}$. Consider the case $\mathcal{B} \cap L \cap B_{c} \neq \emptyset$ (the other case is trivial), and select some condition $b_{o} \in$ $\mathcal{B} \cap L \cap B_{c}$. Let $L^{\prime}$ be the largest subnet of $\Omega_{\mathcal{P}}$ satisfying the following properties:

1. $\mathcal{B} \cup L^{\prime}$ is a branching process.

2. $L^{\prime}$ contains $\left\{b_{o}\right\} \cup b_{o}^{\bullet}$, and $L^{\prime} \cap E \cap \mathcal{B}=\emptyset$ (i.e., $L^{\prime}$ is a non trivial continuation of $\mathcal{B})$.

3. If $e \in L^{\prime}$, then $e^{\bullet} \in L^{\prime}$ (maximal nodes of $L^{\prime}$, if any, must be conditions).

4. If $b \in L^{\prime}, b \neq b_{o}$, and $b \in B_{c}$, then $b^{\bullet} \cap L^{\prime}=\emptyset$ (a branching condition different from $b_{o}$ has no continuation within $L^{\prime}$ ).

First, note that the set of subnets of $\Omega_{\mathcal{P}}$ satisfying the above properties is non empty: since $\mathcal{P}$ is free choice, the subnet $\left\{b_{o}\right\} \cup b_{o}^{\bullet} \cup\left(b_{o}^{\bullet}\right)^{\bullet}$ satisfies these properties. Since, on 
the other hand, this set of subnets is stable under union, it follows that $L^{\prime}$ is well defined. By construction, $\mathcal{B}^{\prime} \triangleq \mathcal{B} \cup L^{\prime}$ is a stopping time, and $\mathcal{B} \neq \mathcal{B}^{\prime} \subseteq \mathcal{B}^{\prime \prime}$. Since $L$ is a layer, this implies $\mathcal{B}^{\prime}=\mathcal{B}^{\prime \prime}$ and thus $L^{\prime}=L$.

\section{Proof of theorem 1}

Consider the DAG $(\mathcal{L}, \prec)$ introduced after Lemma 3, we denote it simply by $\mathcal{L}$ by abuse of notation. Stopping time $\mathcal{B}$ identifies with some prefix $\mathcal{L}^{\mathcal{B}}$ of this DAG, and $\mathcal{B}_{+}$identifies with the corresponding tail $\mathcal{L}^{\mathcal{B}_{+}}$of it (be careful that the present $X_{\mathcal{B}}$ is not encoded in DAG $\mathcal{L}$, since the present is not a union of layers). Define successive slices of $\mathcal{L}^{\mathcal{B}_{+}}$as follows: set

$$
\begin{aligned}
\mathcal{L}_{1}^{\mathcal{B}_{+}} & =\left\{L \in \mathcal{L}: L \notin \mathcal{L}^{\mathcal{B}} \text { and }{ }^{\bullet} L \in \mathcal{L}^{\mathcal{B}}\right\} \\
\forall n \geq 1, \mathcal{L}_{n+1}^{\mathcal{B}_{+}} & =\left\{L \in \mathcal{L}: L \notin \mathcal{L}_{n}^{\mathcal{B}_{+}} \text {and }{ }^{\bullet} L \in \mathcal{L}_{n}^{\mathcal{B}_{+}}\right\}
\end{aligned}
$$

where ${ }^{\bullet} L$ denotes the set of parent nodes in DAG $\mathcal{L}$. Each slice $\mathcal{L}_{n}^{\mathcal{B}_{+}}$uniquely defines a corresponding slice on the unfolding $\Omega_{\mathcal{P}}$ by taking the union of the layers represented in slice $\mathcal{L}_{n}^{\mathcal{B}_{+}}$, we denote by $\mathcal{S}_{n}$ the slice of $\Omega_{\mathcal{P}}$ obtained in this way. Of course, we have $\mathcal{B}_{+}=\bigcup_{n \geq 1} \mathcal{S}_{n}$, and we claim that

$$
\mathcal{F}_{\mathcal{B}}^{+}=\bigvee_{n \geq 1} \mathcal{F}_{\mathcal{S}_{n}}
$$

where $\mathcal{F}_{\mathcal{S}_{n}}$ is defined according to $(17)^{3}$. To show (33), it is enough, by induction, to show that

$$
\mathcal{F}_{\mathcal{B}}^{+}=\mathcal{F}_{\mathcal{S}_{1}} \bigvee \mathcal{F}_{\mathcal{B}^{\prime}}^{+}, \text {where } \mathcal{B}^{\prime}=\mathcal{B} \vee \mathcal{S}_{1}
$$

In the sequel, we write $\mathcal{S}$ for short instead of $\mathcal{S}_{1}$. Property (34) rewrites as follows:

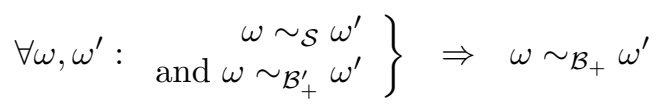

Now, assume that $\omega$ and $\omega^{\prime}$ satisfy $\omega \sim_{\mathcal{S}} \omega^{\prime}$ and $\omega \sim_{\mathcal{B}_{+}^{\prime}} \omega^{\prime}$. Consequently there exist two isomorphisms $\psi$ and $\psi_{+}$of labelled graphs, such that $\omega_{\mathcal{S}}^{\prime}=\psi\left(\omega_{\mathcal{S}}\right)$ and $\omega_{\mathcal{B}_{+}^{\prime}}^{\prime}=\psi_{+}\left(\omega_{\mathcal{B}_{+}^{\prime}}\right)$. Consider the suffix boundary of $\mathcal{S}$, defined by $\mathcal{S}_{+}=\mathcal{S} \cap \mathcal{B}_{+}^{\prime}$. Since $\mathcal{S}$ is a slice, we have

$$
\min \left(\mathcal{B}_{+}^{\prime}\right)=\mathcal{S}_{+} .
$$

We wish to glue together $\psi$ and $\psi_{+}$at $\omega_{\mathcal{S}} \cap \mathcal{S}_{+}=\omega_{\mathcal{S}_{+}}$. However it is not generally true that $\psi$ and $\psi_{+}$agree on $\omega_{\mathcal{S}_{+}}$, so gluing is not immediate in this case. To circumvent this difficulty, we remark that the restrictions $\psi_{/ \mathcal{S}_{+}}$and $\psi_{+} \mathcal{S}_{+}$, of $\psi$ and $\psi_{+}$to $\mathcal{S}_{+}$respectively, both relate $\omega_{\mathcal{S}_{+}}$ and $\omega_{\mathcal{S}_{+}}^{\prime}$ via an isomorphism of labelled graphs. Hence there must exist an automorphism $\chi$ of $\omega_{\mathcal{S}_{+}}$, such that

$$
\psi_{+} / \mathcal{S}_{+} \circ \chi=\psi / \mathcal{S}_{+}
$$

${ }^{3}$ An active reader would probably guess that $\mathcal{F}_{\mathcal{B}}^{+}=\bigvee_{L \subset \mathcal{B}_{+}} \mathcal{F}_{L}$ also holds, but this conjecture is indeed false! The reason is that layers in fact also carry hidden information about their past: for instance the predicate $\omega_{L} \neq \emptyset$ indicates that $\omega_{\mathcal{B}}$ belongs to the set of configurations reaching $L$, and sometimes this set is even a singleton! Slices were introduced to avoid this oddity: all runs traverse all slices. where $\circ$ denotes the composition of maps. Using the definition of branching processes, $\chi$ extends to an automorphism of $\omega_{\mathcal{S}_{+}} \cup\left(\omega_{\mathcal{S}_{+}}\right)^{\bullet}$, and, by induction and using (36), it extends to an automorphism of $\omega_{\mathcal{B}_{+}^{\prime}}$, we call it again $\chi$. But now, $\psi$ and $\psi_{+} \circ \chi$ are two isomorphisms which map $\omega_{\mathcal{S}}$ onto $\omega_{\mathcal{S}}^{\prime}$ and $\omega_{\mathcal{B}_{+}^{\prime}}$ onto $\omega_{\mathcal{B}_{+}^{\prime}}^{\prime}$ respectively, and agree on $\mathcal{S}^{+}$. Hence they can be glued together to form an isomorphism $\psi_{\mathcal{B}_{+}}$, mapping $\omega_{\mathcal{B}_{+}}$onto $\omega_{\mathcal{B}_{+}}^{\prime}$. This proves (35) and hence also (34) and (33).

We are now ready to prove our Markov property. We first prove that, for $A \in \mathcal{F}_{\mathcal{S}}$, the following holds:

$$
\mathbf{P}^{\pi}\left(A \mid \mathcal{F}_{\mathcal{B}}\right)=\mathbf{P}^{\pi}\left(A \mid \mathcal{X}_{\mathcal{B}}\right)
$$

By definition of $\mathcal{F}_{\mathcal{S}}$ it is enough to prove (37) for $A$ a set of the form $A=\left\{v: v \sim_{\mathcal{S}} \omega^{(1)}\right\}$, for $\omega^{(1)}$ a fixed run belonging to $\Omega_{\mathcal{P}}$. For $\omega_{\mathcal{B}}$ a maximal configuration of $\mathcal{B}$, define

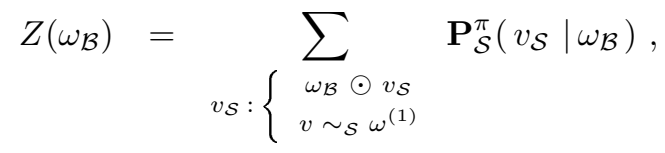

where $\sum_{\emptyset}=0$ by convention, and

$$
\mathbf{P}_{\mathcal{S}}^{\pi}\left(v_{\mathcal{S}} \mid \omega_{\mathcal{B}}\right)=\prod_{\substack{L \in \mathcal{L}_{\mathcal{S}} \\ v_{L} \neq \emptyset}} \mathbf{P}_{L}^{\pi}\left(v_{L} \mid \omega_{\mathcal{B}}\right)
$$

where $\mathbf{P}_{L}^{\pi}$ is defined in (10), and $\mathcal{L}_{\mathcal{S}} \triangleq\left\{L \in \mathcal{L}_{c}: L \subseteq \mathcal{S}\right\}$. Note that $\omega_{\mathcal{B}} \odot v_{L}$ holds in $(39)$, since $\omega_{\mathcal{B}} \odot v_{\mathcal{S}}$ and $L \subset \mathcal{S}$, hence $\mathbf{P}_{L}^{\pi}\left(v_{L} \mid \omega_{\mathcal{B}}\right)$ therein is well defined. We claim the following:

$$
\begin{aligned}
& Z \text { is } \mathcal{X}_{\mathcal{B}}-\text { measurable } \\
& Z=\mathbf{P}^{\pi}\left(A \mid \mathcal{F}_{\mathcal{B}}\right)
\end{aligned}
$$

Note that $(40,41)$ together prove $(37)$. To prove $(40)$ expand $(38,39)$ using $(10)$, this yields

$$
\begin{aligned}
Z\left(\omega_{\mathcal{B}}\right) & =\sum_{v_{\mathcal{S}}:\{} \prod_{\substack{\omega_{\mathcal{B}} \odot v_{\mathcal{S}} \\
v \sim_{\mathcal{S}} \omega^{(1)}}} \frac{1}{v_{L} \neq \mathcal{L}_{\mathcal{S}}} \prod_{C_{L}} \prod_{b \in L_{c} \cap v_{L}} \pi(\varphi(e) \mid \varphi(b))
\end{aligned}
$$

where $e$ is the unique event belonging to $v_{L} \cap b^{\bullet}$. To prove (40) we need to show that:

$$
\omega \sim_{X_{\mathcal{B}}} \omega^{\prime} \Rightarrow Z\left(\omega_{\mathcal{B}}\right)=Z\left(\omega_{\mathcal{B}}^{\prime}\right)
$$

Set $M=\varphi\left(\max \left(\omega_{\mathcal{B}}\right) \cap X_{\mathcal{B}}\right)$ and $M^{\prime}=\varphi\left(\max \left(\omega_{\mathcal{B}}^{\prime}\right) \cap X_{\mathcal{B}}\right) . M$ and $M^{\prime}$ are the live submarkings reached by $\omega$ and $\omega^{\prime}$, respectively, when exiting $\mathcal{B}$. Note that, since $\max \left(\omega_{\mathcal{B}}\right) \backslash X_{\mathcal{B}}$ (resp. $\left.\max \left(\omega_{\mathcal{B}}^{\prime}\right) \backslash X_{\mathcal{B}}\right)$ do not belong to $\mathcal{B}_{+}$, the corresponding submarkings are blocked for ever. Assume $\omega \sim_{X_{\mathcal{B}}} \omega^{\prime}$, this implies $M=M^{\prime}$. For $c$ a co-set, denote by $\Omega_{\mathcal{P}}^{c}$ the subnet of unfolding $\Omega_{\mathcal{P}}$ containing all nodes $x$ such that $x \succ c$, i.e., $\Omega_{\mathcal{P}}^{c}$ is the "future" of $c$. Take in particular $c=\max \left(\omega_{\mathcal{B}}\right) \cap X_{\mathcal{B}}$ and $c^{\prime}=\max \left(\omega_{\mathcal{B}}^{\prime}\right) \cap X_{\mathcal{B}}$. Since $M=M^{\prime}$, it follows that $\Omega_{\mathcal{P}}^{c}$ and $\Omega_{\mathcal{P}}^{c^{\prime}}$ are isomorphic, when 
seen as labelled graphs. But the $v_{\mathcal{S}}$ 's defined in the right hand side of formula (42), for the two cases of expanding $Z\left(\omega_{\mathcal{B}}\right)$ and $Z\left(\omega_{\mathcal{B}}^{\prime}\right)$, are all subsets of $\Omega_{\mathcal{P}}^{c}$ and $\Omega_{\mathcal{P}}^{c^{\prime}}$, respectively. And the same holds for the sets of branching conditions defined in the right hand side of formula (42). Since, on the other hand, the terms $\pi(\varphi(e) \mid \varphi(b))$ involved in these expansion are invariant under an isomorphism of labelled occurrence nets, we deduce that $Z\left(\omega_{\mathcal{B}}\right)=Z\left(\omega_{\mathcal{B}}^{\prime}\right)$ holds, this proves (43).

Let us prove (41). We need to prove that

$$
\forall A^{\prime} \in \mathcal{F}_{\mathcal{B}}, \mathbf{P}^{\pi}\left(A^{\prime} \cap A\right)=\mathbf{E}^{\pi}\left(\mathbf{1}_{A^{\prime}} Z\right),
$$

where $\mathbf{E}^{\pi}$ denotes expectation with respect to $\mathbf{P}^{\pi}$, and $\mathbf{1}_{A^{\prime}}$ is the indicator function of set $A^{\prime}$. It is enough to prove (44) for $A^{\prime}=\left\{v: v \sim_{\mathcal{B}} \omega^{(0)}\right\}$, where $\omega^{(0)}$ is fixed. But then, using explicit formulas $(10,11)$, we get

$$
\begin{aligned}
& \mathbf{P}^{\pi}\left(\left\{v:\left(v \sim_{\mathcal{B}} \omega^{(0)}\right) \wedge\left(v \sim_{\mathcal{S}} \omega^{(1)}\right)\right\}\right) \\
= & \mathbf{P}_{\mathcal{B}}^{\pi}\left(\omega_{\mathcal{B}}^{(0)}\right) \times \sum_{\substack{\omega_{\mathcal{B}}^{(0)} \odot v_{\mathcal{S}} \\
v \sim_{\mathcal{S}} \omega^{(1)}}} \mathbf{P}_{\mathcal{S}}^{\pi}\left(v_{\mathcal{S}} \mid \omega_{\mathcal{B}}^{(0)}\right) \\
= & \int_{\left(v \sim_{\mathcal{B}} \omega^{(0)}\right)} Z\left(\omega_{\mathcal{B}}^{(0)}\right) d \mathbf{P}^{\pi}(w),
\end{aligned}
$$

which proves (44). Finally, formula (37) implies, by usual induction, our expected Markov property.

\section{Proof of theorem 3}

Consider the two conditions (i,ii) of Lemma 5, which involve the unfolding $\widehat{\Omega}_{\mathcal{P}}$.

Denote by $\widehat{c}$ the set of maximal conditions of $\widehat{\omega}$, this coset represents some sub-marking $\widehat{m}$ of $\widehat{\mathcal{P}}$. Sub-marking $\widehat{m}$ is composed of dummy places and is dead, meaning that its postset is empty. Next, denote by $\widehat{c}^{\prime \prime}$ the set of maximal conditions of $\widehat{\omega} \cap \widehat{\omega}^{\prime}$, i.e., the co-set at which the two configurations $\widehat{\omega}$ and $\widehat{\omega}^{\prime}$ branch. This co-set represents some sub-marking $\widehat{m}^{\prime \prime}$ of $\widehat{\mathcal{P}}$. Complement co-set $\widehat{c}^{\prime \prime}$ into a cut $\widehat{\mathbf{c}}^{\prime \prime}$ contained in $\widehat{\omega} \cap \widehat{\omega}^{\prime}$, having no dummy conditions, and representing the marking $\widehat{M}^{\prime \prime}$. Sub-marking $\widehat{m}^{\prime \prime}$ possesses no dummy place, and sub-marking $\widehat{m}$ is reachable from marking $\widehat{M}^{\prime \prime}$. Focus on $\widehat{\omega}^{\prime}$, and denote by $\widehat{\mathcal{M}}^{\prime}$ the set of markings traversed by $\widehat{\omega}^{\prime}$ after reaching $\widehat{M}^{\prime \prime}$. Then $\widehat{\mathcal{M}}^{\prime}$ contains no dead submarking composed of dummy places.

To summarize, conditions (i,ii) of Lemma 5 are equivalent to the following condition, which can be checked using the doubly-complete prefix of $\widehat{\mathcal{P}}$ :

Condition 1: There exist a reachable marking $\widehat{M}^{\prime \prime}$, a sub-marking $\widehat{m}$ reachable from $\widehat{M}^{\prime \prime}$, and a firing sequence $\widehat{\mathcal{M}}^{\prime}$ starting from $\widehat{M}^{\prime \prime}$, such that: $1 / \widehat{M}^{\prime \prime}$ contains no dummy place, $2 / \widehat{m}$ is dead and composed of dummy places, and $3 / \widehat{\mathcal{M}}^{\prime}$ contains no dead submarking composed of dummy places.

\section{Proof of theorem 4}

Since co-set $c$ is reachable from any marking, it is a recurrent set, meaning that almost every infinite configuration of $\widehat{\Omega}_{\mathcal{P}}$ contains infinitely many copies of co-set $c$. Denote by $\widehat{\Omega}_{n}$ the subset of $\widehat{\Omega}_{\mathcal{P}}$ composed of the configurations that have crossed at least $n$ times co-set $c$. We have

$$
\Psi^{-1}\left(\Omega_{\mathcal{P}}\right) \subseteq \limsup _{n \rightarrow \infty} \widehat{\Omega}_{n}
$$

Let $\alpha>0$ be the probability that the choices performed at co-set $c$ result in a blocking. We have $\widehat{\mathbf{P}}^{\pi}\left(\Omega_{n}\right) \leq$ $(1-\alpha) \widehat{\mathbf{P}}^{\pi}\left(\Omega_{n-1}\right)$, whence

$$
\sum_{n} \widehat{\mathbf{P}}^{\pi}\left(\Omega_{n}\right) \leq \sum_{n}(1-\alpha)^{n}<+\infty .
$$

From (45) and (46), and by the Borel-Cantelli lemma, we get that

$$
\mathbf{P}^{\pi}\left(\Omega_{\mathcal{P}}\right)=\widehat{\mathbf{P}}^{\pi}\left(\Psi^{-1}\left(\Omega_{\mathcal{P}}\right)\right)=0 .
$$

This proves the theorem.

AcKnowledgement. The authors wish to thank Samy Abbes for pointing out mistakes in a draft version of this article.

\section{REFERENCES}

[1] A. Benveniste, E. Fabre, and S. Haar. "Markov nets: probabilistic models for distributed and concurrent systems". Irisa Research Report 1538, May 2003. Extended version of this paper ftp://ftp.irisa.fr/techreports/2003/PI-1538.ps.gz

[2] A. Aghasaryan, E. Fabre, A. Benveniste, R. Boubour, C. Jard. Fault detection and diagnosis in distributed systems : an approach by partially stochastic Petri nets, Discrete event dynamic systems: theory and application, special issue on Hybrid Systems, vol. 8, pp. 203-231, June 1998.

[3] M. Ajmone Marsan, G. Balbo, G. Conte, S. Donatelli and G. Franceschinis. Modeling with Generalized Stochastic Petri nets, Wiley series in parallel computing, 1995.

[4] M. Ajmone Marsan, G. Balbo, G. Chiola, and G. Conte. Generalized Stochastic Petri Nets Revisited: Random Switches and Priorities. Proc. PNPM '87, IEEE-CS Press, pp. 44-53.

[5] F. Baccelli, S. Foss, and B. Gaujal. Free choice Petri nets - an algebraic approach. IEEE Trans. on Autom. Control, 41(12), 1751-1778, Dec. 1996.

[6] F. Bause and P.S. Kritzinger. Stochastic Petri Nets, An introduction to the Theory. Verlag Vieweg, 1996.

[7] A. Benveniste, B.C. Levy, E. Fabre, P. Le Guernic, "A Calculus of Stochastic Systems: specification, simulation, and hidden state estimation," Theoretical Computer Science, no. 152, pp. 171-217, 1995.

[8] A. Benveniste, E. Fabre, S. Haar, C. Jard. Diagnosis of asynchronous discrete event systems, a net unfolding approach. To appear in IEEE Trans. on Autom. Control, May 2003.

[9] C. Cassandras and S. Lafortune. Introduction to discrete event systems. Kluwer Academic Publishers, 1999.

[10] R. David and H. Alla. Petri nets for Modeling of Dynamical Systems - a Survey, Automatica, 30(2), 175-202, 1994.

[11] J. Desel, and J. Esparza. Free Choice Petri Nets. Cambridge University Press, 1995.

[12] R. Debouk, S. Lafortune, and D. Teneketzis. Coordinated decentralized protocols for failure diagnosis of discrete event systems. Discrete Event Dynamic Systems: theory and application. $10(1 / 2), 33-86,2000$.

[13] J. Engelfriet. Branching Processes of Petri Nets. Acta Informatica 28, 1991, pp 575-591.

[14] J. Esparza, S. Römer, and W. Vogler. An improvement of McMillan's unfolding algorithm. Formal Methods in System Design 20(3):285-310, May 2002.

[15] J. Esparza, and S. Römer. An unfolding algorithm for synchronous products of transition systems, in proceedings of $C O N$ CUR'99, LNCS 1664, Springer Verlag, 1999.

[16] E. Fabre, A. Benveniste, C. Jard, L. Ricker, and M. Smith. Distributed state reconstruction for discrete event systems. Proc. of 
the 2000 IEEE Control and Decision Conference (CDC), Sydney, Dec. 2000.

[17] S. Haar. Branching processes of general S/T-Systems. Proc. Workshop on Concurrency, MFCS'98, Brno. Electronic Notes in Theoretical Computer Science vol. 18, Elsevier, 1998. http://www.elsevier.nl/locate/entcs/volume18.html

[18] S. Haar. Occurrence Net Logics. Fundamenta Informaticae 43, pp 105-127, 2000.

[19] S. Haar. Probabilistic Unfoldings and Partial Order Fairness in Petri Nets. In: H. Hermanns and R. Segala (eds.), Process Algebra and Probabilistic Methods. Proceedings PAPM-ProbMiV 2002, LNCS 2399, 95-114.

[20] S. Haar. Probabilistic Cluster Unfoldings. Fundamenta Informaticae 53(3-4), 281-314, 2002.

[21] Peter J. Haas. Stochastic Petri Nets. Modeling, Stability, Simulation. Springer Verlag, Springer Series in Operations Research, July 2002 .

[22] K.X. He and M.D. Lemmon. Liveness verification of discreteevent systems modeled by $n$-safe Petri nets. in Proc. of the 21st Int. Conf. on Application and Theory of Petri Nets, Aarhus, June 2000.

[23] K.X. He and M.D. Lemmon. On the existence of livenessenforcing supervisory policies of discrete-event systems modeled by $n$-safe Petri nets. in Proc. of IFAC'2000 Conf. on Control Systems Design, special session on Petri nets, Slovakia, June 2000.

[24] K. McMillan. Symbolic model checking: an approach to the state explosion problem. Kluwer, 1993.

[25] P.A. Meyer and C. Dellacherie. Probabilités et potentiels, chap. I-IV, Hermann, Paris, ISBN 270561372 2, 1975.

[26] M. Nielsen, G. Plotkin, and G. Winskel. Petri nets, event structures, and domains. Part I. Theoretical Computer Science 13:85-108, 1981

[27] M. Raynal. Networks and Distributed Computation: concepts, tools and algorithms. The MIT Press, 1988, 166 pages. (ISBN $0-262-18130-4)$

[28] W. Reisig. Petri nets. Springer Verlag, 1985.

[29] G. Shafer. A mathematical theory of evidence. Princeton University Press, Princeton NJ, 1976.

[30] H. Völzer. Randomized non-sequential processes. Proceedings CONCUR $2001-12$ th Int. Conf. on Concurrency Theory, Aalborg. LNCS 2154, 184-201, Springer Verlag, August 2001.

[31] H. Völzer. Fairness, Randomisierung und Konspiration in verteilten Algorithmen. PhD thesis, Humboldt-Universität zu Berlin, Institut für Informatik, feb. 2001, in german. 